\title{
Combined Metabolic Activators Improves Cognitive Functions in Alzheimer's Disease
}

Burak Yulug, ${ }^{1, \#}$, Ozlem Altay ${ }^{2, \#}$, Xiangyu $\mathrm{Li}^{2}{ }^{2, \#}$, Lutfu Hanoglu ${ }^{3}$, Seyda Cankaya ${ }^{1}$, Simon Lam $^{4}$, Hong Yang ${ }^{2}$, Ebru Coskun ${ }^{3}$, Ezgi İdil ${ }^{1}$, Rahim Nogaylar ${ }^{1}$, Cemil Bayram ${ }^{5}$, Ismail Bolat $^{6}$, Sena Öner ${ }^{7}$, Özlem Özdemir Tozlu ${ }^{7}$, Mehmet Enes Arslan ${ }^{7}$, Ahmet Hacımuftuoglu ${ }^{5}$, Serkan Yildırım ${ }^{6}$, Muhammad Arif ${ }^{2}$, Saeed Shoaie ${ }^{2,4}$, Cheng Zhang ${ }^{2.8}$, Jens Nielsen ${ }^{9}$, Hasan Turkez $^{10}$, Jan Borén ${ }^{11}$, Mathias Uhlén ${ }^{2, *}$, Adil Mardinoglu ${ }^{2,4, *,+}$

${ }^{1}$ Department of Neurology and Neuroscience, Faculty of Medicine, Alanya Alaaddin Keykubat University, Antalya, Turkey

${ }^{2}$ Science for Life Laboratory, KTH - Royal Institute of Technology, Stockholm, Sweden

${ }^{3}$ Department of Neurology, Faculty of Medicine, Istanbul Medipol University, Istanbul, Turkey

${ }^{4}$ Centre for Host-Microbiome Interactions, Faculty of Dentistry, Oral \& Craniofacial Sciences, King's College London, London, United Kingdom

${ }^{5}$ Department of Medical Pharmacology, Faculty of Medicine, Atatürk University, Erzurum, Turkey.

${ }^{6}$ Department of Pathology, Veterinary Faculty, Ataturk University, Erzurum, Turkey

${ }^{7}$ Department of Molecular Biology and Genetics, Faculty of Science, Erzurum Technical University, Erzurum, Turkey

${ }^{8}$ School of Pharmaceutical Sciences, Zhengzhou University, Zhengzhou, PR China

${ }^{9}$ Department of Biology and Biological Engineering, Chalmers University of Technology, Gothenburg, Sweden

${ }^{10}$ Department of Medical Biology, Faculty of Medicine, Atatürk University, Erzurum, Turkey

${ }^{11}$ Department of Molecular and Clinical Medicine, University of Gothenburg and Sahlgrenska University Hospital, Gothenburg, Sweden

*Correspondence: adilm@scilifelab.se; mathias.uhlen@scilifelab.se

+Lead author: Adil Mardinoglu (adilm@scilifelab.se)

Emails: burakyulug@gmail.com; havva.altay@scilifelab.se; xiangyu.li@scilifelab.se;

lhanoglu@kure.com.tr; cankayaseyda@ hotmail.com; simon.1.lam@kcl.ac.uk; hong.yang@scilifelab.se; ebrucosskun@gmail.com; ezgi.idil92@gmail.com; ngylrrhm@gmail.com; cemil489@gmail.com; ismail.bolat@ atauni.edu.tr; senaoner02@gmail.com; ozlem.ozdemir@erzurum.edu.tr; enesiyte@gmail.com; ahmeth@atauni.edu.tr; syildirim@atauni.edu.tr; muhammad.arif@scilifelab.se; saeed.shoaie@scilifelab.se; cheng.zhang@scilifelab.se; nielsenj@chalmers.se; 
medRxiv preprint doi: https://doi.org/10.1101/2021.07.14.21260511; this version posted August 15, 2021. The copyright holder for this preprint

hasanturkez@yahoo.com; Jan.Boren@wlab.gu.se; mathias.uhlen@ scilifelab.se; adilm@scilifelab.se

\section{ABSTRACT}

Alzheimer's disease (AD) is associated with metabolic abnormalities linked to critical elements of neurodegeneration. Here, we analysed the brain transcriptomics data of more than $600 \mathrm{AD}$ patients using genome-scale metabolic models and provided supporting evidence of mitochondrial dysfunction related to the pathophysiologic mechanisms of AD progression. Subsequently, we investigated, in a rat model of $\mathrm{AD}$, the oral administration of Combined Metabolic Activators (CMAs), consisting of NAD+ and glutathione precursors, to explore the effect for improvement of biological functions in AD. CMAs includes L-serine, nicotinamide riboside, N-acetyl-L-cysteine, and L-carnitine tartrate, salt form of L-carnitine. The study revealed that supplementation of the CMAs improved the AD-associated histological parameters in the animals. Finally, we designed a randomized, double-blinded, placebocontrolled human phase 2 clinical trial and showed that the administration of CMAs improves cognitive functions in $\mathrm{AD}$ patients. As decreased $\mathrm{AD}$ Assessment Scale-cognitive subscale (ADAS-Cog) score is the indicator of the improved cognitive function in AD patients, we observed a significant decrease of ADAS-Cog scores on Day 84 vs Day 0 ( $\log 2 \mathrm{FC}=-0.37$, (29\% improvement), p-value $=0.00001)$ in the CMA group. We also observed a significant decrease in the placebo group on Day 84 vs Day $0(\log 2 \mathrm{FC}=-0.19,(14 \%$ improvement $), \mathrm{p}$ value $=0.001)$ due to the recommendations of exercise and Mediterranean diet to all $\mathrm{AD}$ patients participated in the trial. A comprehensive analysis of the human plasma metabolome and proteome revealed that plasma levels of proteins and metabolites associated with redox metabolism are significantly improved after treatment. In conclusion, our results show that treating $\mathrm{AD}$ patients with $\mathrm{CMAs}$ leads to enhanced cognitive functions, suggesting a role for such a therapeutic regime in treating $\mathrm{AD}$ and other neurodegenerative diseases. 


\section{HIGHLIGHTS:}

- $\quad$ Brain transcriptomics data of more than $600 \mathrm{AD}$ patients is analysed.

- $\quad$ Performed an in vivo study using Combined Metabolic Activators (CMAs) in AD rat models.

- We performed a randomized, double-blinded, placebo-controlled human phase 2 clinical trial.

- We showed that cognitive functions in AD patients is improved $29 \%$ in the CMA group whereas $14 \%$ in the placebo group.

\section{KEYWORDS:}

Alzheimer's disease: Combined Metabolic Activators (CMAs), Multi-omics analysis; Systems Biology; Systems Medicine 
medRxiv preprint doi: https://doi.org/10.1101/2021.07.14.21260511; this version posted August 15, 2021. The copyright holder for this preprint

\section{INTRODUCTION:}

Alzheimer's disease (AD) is characterized by progressive synaptic and axonal dysfunction, neuronal loss and cognitive decline (1). There is growing evidence that $\mathrm{AD}$ is closely associated with metabolic and oxidative stress linked to critical elements of neurodegeneration, such as mitochondrial dysfunctions and bioenergetic impairments $(2,3)$. Indeed, increasing data indicate that systemic metabolic disorders, such as insulin resistance, are strongly associated with bioenergetic failure of nerve cells $(4,5)$. This can manifest as cognitive impairment and brain-specific neuropathology while sharing common pathogenic mechanisms with $\mathrm{AD}$, such as impaired glucose metabolism, increased oxidative stress, insulin resistance, and amyloidogenesis $(4,6,7)$. Recent evidence accordingly suggests that patients with type 2 diabetes mellitus are at increased risk of developing $\mathrm{AD}(6)$.

Although the disease is still defined by the accumulation of abnormal amyloid and tau proteins $(8)$, the mechanistic assumption of linear causality between the amyloid cascade and cognitive dysfunction in $\mathrm{AD}$ is flawed, since amyloid-lowering approaches have failed to provide cognitive benefits in human clinical trials (9). A growing body of evidence suggests that impaired brain energy metabolism in $\mathrm{AD}$ may contribute to cognitive decline. At the same time, therapeutic options, such as drugs typically prescribed for metabolic disorders that improve metabolic status, may slow cognitive decline or prevent dementia progression (10). This is suggested by positron emission tomography imaging studies revealing baseline cerebral glucose metabolism abnormalities before the onset of cognitive symptoms in patients with $\mathrm{AD}(11)$. In addition, recent preclinical data indicate that ageing and $\mathrm{AD}$ are associated with the reorganization of brain energy metabolism, including an overall increase in lactate secretion and the downregulation of bioenergetic enzymes $(12,13)$.

Although current research is paving the way for developing neuroprotective therapeutics, the results of early clinical trials of drugs targeting single pathways have been mostly unsuccessful. A divergent approach combining multiple compounds that simultaneously reduce oxidative injury and improve bioenergetics, in other words, targeting various pathways has been proposed as a therapeutic strategy associated more likely with successful translational outcomes (14). Previous research identified limited serine availability, reduced de novo glutathione synthesis, and altered NAD+ metabolism based on the combining multiomics profiling of transgenic $\mathrm{AD}$ mouse model of $\mathrm{AD}$ (15). Consistent with this, it also has been reported that age- and $\mathrm{AD}$-associated metabolic shifts responded well to 
$\mathrm{NAD}(\mathrm{P})+/ \mathrm{NAD}(\mathrm{P}) \mathrm{H}$ redox-dependent reactions $(16,17)$. These findings were confirmed by human metabolomic data showing significantly altered cerebrospinal fluid (CSF) acylcarnitine levels in patients with $\mathrm{AD}$, which correlated with the decline of cognitive functions and structural brain abnormalities $(18,19)$.

Based on integrative network analysis of non-alcoholic fatty liver disease multi-omics data, we have developed a mixture combined metabolic activators (CMAs) consisting of L-serine, $\mathrm{N}$-acetyl cysteine (NAC), nicotinamide riboside (NR), and L-carnitine tartrate (LCAT, the salt form of L-carnitine) and showed that administration of CMAs activates mitochondria, improves inflammation markers in animals and humans (20-24). We have also found that the administration of CMAs promotes mitochondrial fatty acid uptake from the cytosol, facilitates the fatty acid oxidation in the mitochondria, and alleviates oxidative stress (25). Recently, we reported that CMAs administration effectively increased fatty acid oxidation and de novo glutathione generation, as evidenced by metabolomic and proteomic profiling (20). Moreover, plasma levels of metabolites associated with antioxidant metabolism and inflammatory proteins were improved in COVID-19 patients treated with CMAs compared to the placebo (24).

Here, we first analysed brain transcriptomics data obtained from 629 AD patients and 704 control subjects using genome-scale metabolic models and revealed that mitochondrial dysfunction is involved in the underlying molecular mechanisms associated with AD. Second, we tested the effect of CMAs, which has been shown to activate mitochondria in the AD rat models and showed that supplementation of the CMAs improved the $\mathrm{AD}$ and associated functions in animals. Next, we hypothesized that $\mathrm{AD}$ patients could be treated with the administration of the CMAs by activating the mitochondria in the brain tissue of the patients. Finally, we designed a randomized, double-blinded, placebo-controlled human phase 2 clinical study, studied the effect of administration on the global metabolism of AD patients and showed that administration of CMAs improves the cognitive functions in AD patients. 
medRxiv preprint doi: https://doi.org/10.1101/2021.07.14.21260511; this version posted August 15, 2021. The copyright holder for this preprint

\section{RESULTS}

\section{Analysis of transcriptomics data reveals mitochondrial dysfunction in the brain of AD patients.}

To identify the metabolic dysregulations based on brain transcriptomics data, we obtained the global mRNA expression profiling of 629 AD patients and 704 controls from the Religious Orders Study/Memory and Aging Project (ROSMAP) (26-28). We performed differential expression analysis and identified 914 significantly (p-adjusted $<1.0$ E-10) upregulated and 1725 significantly (p-adjusted < 1.0 E-10) downregulated differentially expressed genes (DEGs) (Figure 1A, Dataset S1). We identified several upregulated metabolism-related genes, including pyruvate dehydrogenase kinase 4 (PDK4), carnitine palmitoyl transferase (CPT1A), hexokinase 2 (HK2), and spermine oxidase (SMOX), as well as downregulated genes including acyl-CoA dehydrogenases (ACADs), ATP synthase (ATP5MGL), and acetyl- and acyltransferases (GCNT7, MBOAT4, GALNT17). These results suggest that there may be some alterations associated with glycolysis, fatty acid biogenesis and the urea cycle in AD compared to control.

The gene set enrichment (GSE) analysis revealed that these DEGs were significantly enriched in the protein synthesis, ATP synthesis, lipid metabolism, cell cycle, cell migration, cell differentiation and cell adhesion pathways (Figure 1B, Dataset S1). Then, we performed reporter metabolite analysis to predict the significantly changed metabolites in each subcellular compartment using a genome-scale metabolic model of brain tissue $(29,30)$. We identified numerous reporter metabolites related to glycolysis, amino acid metabolism (e.g. glycine, serine, threonine, alanine and branched-chain amino acid valine, leucine and isoleucine), mitochondrial metabolism (acyl-CoA and ferredoxin) and TCA cycle (e.g. succinate and glutamine) (Figure 1C and 1D, Dataset S1). These results suggested a widespread perturbation in energy metabolism related to brain cell survival and mitochondrial dysfunction in the brain.

\section{Administration of CMAs to animal models of AD}

To test the effect of CMAs in animals, we provided individual metabolic activators and CMAs to the rat model of $\mathrm{AD}$ which has been developed after intracerebroventricularstreptozotocin (STZ) injection. We observed that administration of all constituents of CMAs (in combination or separately) significantly $(\mathrm{p}<0.05)$ decreased the plasma levels of triglycerides (TG) compared to the control group (Group 4; Figure 2A, Dataset S2). In 
medRxiv preprint doi: https://doi.org/10.1101/2021.07.14.21260511; this version posted August 15, 2021. The copyright holder for this preprint

parallel, administration of only serine or NR significantly decreased total cholesterol $(\mathrm{p}=0.01)$ and low-density lipoprotein (LDL; p=0.03) in rats (Figure 2B, Dataset S2). Additionally, a significant reduction in total cholesterol $(\mathrm{p}=0.04)$ and LDL $(\mathrm{p}=0.03)$ was observed with NACtreated and LCAT-treated rats, respectively (Figure 2B, Dataset S2). Of note, we found significant reductions in plasma AST $(\mathrm{p}=0.03)$ and an increase in plasma ALP ( $\mathrm{p}=0.04)$ concentrations only in LCAT-treated rats (Group 8) (Figure 2B, Dataset S2).

Additionally, the histological analyses and immunofluorescence imaging techniques showed a significant neuronal tissue damage in the high fat diet (HFD) and HFD+STZ groups' brains compared to those of the chow diet group (Figure 2C, Dataset S2). Specifically, the HFD groups developed more hyperaemia as well as more degeneration and necrosis in neurons (Figure 2C, Dataset S2). In parallel, DNA damage markers (namely 8-OHdG and H2A.X) and caspase 3 were elevated in the HFD groups (Figure 2C, Dataset S2). These animal models allowed us to examine each rat group's histopathological differences and assess the brain tissue response to CMAs administration compared to the HFD+STZ group (Figure 2C, Dataset S2). We observed that hyperemia, degeneration and necrosis in neurons were improved by serine, LCAT, or NR supplementation individually as well as in combination. However, we observed a better improvement after the administration of CMAs compared to individual metabolic activators. These findings were also supported by immunohistochemical evidence of decreased immunoreactivity seen in neurons (Figure 2C, Dataset S2). Of note, rats receiving combination therapy (consisting of serine, NAC, LCAT and NR) developed less hyperaemia in brain tissue and no necrosis in neurons (Figure 2C, Dataset S2). In parallel to the improvement in the brain, we also observed dramatic improvement in the liver after the supplementation of CMAs. Scoring of histopathological, immunohistochemical and immunofluorescence findings for brain and liver tissues are presented in Table 1.

\section{CMAs Improves Cognition and Blood Parameters in Alzheimer's Disease Patients}

To test the effect of the CMAs in $\mathrm{AD}$ patients, we performed a double-blind, randomized, placebo-controlled phase 2 study and screened 89 adults diagnosed with AD. We recruited 69 patients older than 50 years with mild to moderate $\mathrm{AD}$ according to ADAS-Cog (AD Assessment Scale-cognitive subscale; $A D A S \geq 12$ ) and the Clinical Dementia Rating Scale Sum of Boxes (CDR-SOB; CDR $\leq 2$ ). Of the 69 patients, 47 were randomly assigned to the CMA group and 22 to the placebo group and completed visit 2 after 28 days. Of these patients, 60 (40 in the CMA group and 20 in the placebo group) completed visit 3 after 84 
medRxiv preprint doi: https://doi.org/10.1101/2021.07.14.21260511; this version posted August 15, 2021. The copyright holder for this preprint

days (Figure 3A, Dataset S3). We assessed the clinical variables on Days 0, 28 and 84, and analysed the differences between the time points in the CMA and placebo groups (Dataset S4).

The patients' mean age participated in the study was 70.8 years (56-86 years), and $52.1 \%$ were men (Dataset S4). The baseline demographic and clinical characteristics were similar in the CMA and placebo groups (Dataset S4). Regarding safety, no severe adverse events occurred and 4 patients $(5.8 \%)$ reported adverse events. All had a mild rash on the body and decided to complete the study (Dataset S3).

We measured clinical variables in all patients and analysed the differences before and after administration in the active and placebo groups (Figure 3B, Dataset S4). As decreased ADASCog score is the indicator of the improved cognitive function in AD patients, the ADAS-Cog scores is significantly decreased on Day 28 vs Day 0 (Log2FoldChange $(\mathrm{FC})=-0.33,(26 \%$ improvement), p-value=0.0000003) and further decreased on Day 84 vs Day $0(\log 2 \mathrm{FC}=-$ 0.37, (29\% improvement), p-value=0.00001) in the CMA group. A slightly but significant improvement is also found in the placebo group on Day 28 vs Day $0(\log 2 \mathrm{FC}=-0.16,(12 \%$ improvement), $\mathrm{p}$-value $=0.009)$ and Day 84 vs Day $0(\log 2 \mathrm{FC}=-0.19,(14 \%$ improvement $), \mathrm{p}$ value $=0.001)$ due to the recommendations of exercise and Mediterranean diet to all $\mathrm{AD}$ patients participated in the trial.

We also analysed the differences between clinical parameters by stratifying the patients into low-scored (mild patients) and high-scored (severe patients) ADAS-Cog groups (>20ADASCog score is high, $\leq 20$ is low). More interestingly, we found a significant improvement of ADAS-Cog scores between Day 28 vs Day 0 (Log2FC $=-0.31$, (24\% improvement), pvalue $=0.002)$ and Day 84 vs Day $0(\log 2 \mathrm{FC}=-0.38,(30 \%$ improvement $), p$-value $=0.003)$ in the severe CMA group and no significance difference in the severe placebo ( $>0.05$ in both time points) group (Figure 3B, Dataset S4). As shown on Figure 3B, we observed a significant difference in the baseline value distribution and mean of ADAS-Cog scores in the severe (ADAS-COG > 20) CMA and placebo group due to the randomization of the subjects. To verify our results, we selected 10 patients from the CMA group with matched ADAS-COG values to the placebo group (P-Value: 0.693) and presented the ADAS-Cog scores in Figure 3C. We recalculated the differences in ADAS-COG scores, and again found significant improvement in the CMA group, whereas no significant difference in the placebo group. Our 
medRxiv preprint doi: https://doi.org/10.1101/2021.07.14.21260511; this version posted August 15, 2021. The copyright holder for this preprint

results indicated that the $\mathrm{AD}$ patients with high $\mathrm{ADAS}-\mathrm{Cog}$ scores are more responsive to CMA.

Analysis of secondary outcome variables showed that serum alanine aminotransferase (ALT) levels ( $\log 2 \mathrm{FC}=-0.38$, (30\% improvement), $\mathrm{p}$-value=0.01) and the uric acid levels $(\log 2 \mathrm{FC}=-0.19,(14 \%$ improvement $), \mathrm{p}$-value=0.001) were significantly lower on Day 84 vs Day 0 only in the CMA group (Figure 3D, Dataset S4). This reduction was seen both in highand low-ALT level groups. In contrast, we found that no significantly altered parameters on Day 84 vs Day 0 in the placebo group (Figure 3D, Dataset S4). Hence, we observed that the ALT level and uric acid were significantly improved due to the administration of CMAs as previously reported in the Phase 2 NAFLD and Phase 3 Covid-19 clinical trials $(24,31)$.

We also measured the level of complete blood count parameters and found that their levels were significantly changed in the CMA group (Figure 3D, Dataset S4). We found that the levels of platelets, basophil\% and absolute numbers of basophil and neutrophil were significantly lower on Day 84 vs Day 0 only in the CMA group. In contrast, we found that the levels of monocytes were significantly increased on Day 84 vs Day 0 in the CMA group (Figure 3D, Dataset S4). Hence, our analysis indicated that the administration of CMAs improved the clinical parameters in parallel to the improvement in cognitive functions in $\mathrm{AD}$ patients.

\section{Blood profile informs the response to CMAs}

Treatment response variability and clinical heterogeneity in AD are well documented in the literature. We observed interindividual variability in responses to CMAs as well as in clinical measures. Therefore, we hypothesized that some of the patients would respond better to CMAs than others and that clinical measurements could define these subsets.

To determine whether alanine transferase (ALT), a marker for liver damage, could indicate a better response to CMAs, we stratified the patients into high and low ALT groups by the median value of ALT of all patients on Day 0. As shown in Figure 4A, the patients of the CMA group with low ALT achieved a significant improvement in ADAS-Cog score over different time points, while the patients in the placebo group had no improvement. In contrast, the patients of the CMA group with high ALT levels also exhibited an improved (i.e., decreased) ADAS-Cog score, but the degree of change was not as much as the patients in the CMA group with low ALT levels. Moreover, patients in the placebo group with high ALT 
medRxiv preprint doi: https://doi.org/10.1101/2021.07.14.21260511; this version posted August 15, 2021. The copyright holder for this preprint

levels also achieved an improved ADAS-Cog score. Thus, these results suggest that the patients with low ALT levels are more responsive to CMAs.

We repeated this stratification for each blood parameter to determine the patient conditions in which CMAs produces the greatest response (Figure 4B). In addition to low ALT, we identified high ALP, low GGT, high HCT, high HbA1c, high insulin, high uric acid, high ADAS-Cog, high basophil count, and high red blood cell count as indicators for better responsiveness to CMAs. We also found that individuals who do not drink alcohol or smoke also respond better to CMAs.

\section{CMAs Increases the Plasma Levels of Metabolites Associated with Metabolic Activators}

We first analysed the plasma levels of serine, carnitine, NR and cysteine, and their byproducts. CMAs administration increased the plasma levels of metabolic activators on Day 84 vs Day 0 in the CMA group (Figure 5A, Dataset S5). Moreover, the plasma levels of NR, 1methylnicotinamide, nicotinurate, N1-methyl-2-pyridone-5-carboxamide and nicotinamide (associated with NR and NAD+ metabolism); of serine, glycine and sarcosine (associated with serine and glycine metabolism); and of deoxycarnitine and carnitine (associated with carnitine metabolism) were significantly higher in the CMA group on Day 84 compared to Day 0.

Next, we investigated the relationship with the plasma level of administrated metabolic activators and other metabolites. We analysed 195 of the most significantly correlated plasma metabolites with serine, L-carnitine, NR, and cysteine (Dataset S6). We found two clusters of metabolites that are significantly correlated with cysteine only or together with serine, carnitine and NR. We observed that cysteine had a different plasma changes compared to the other three metabolic activators as has been reported in previous clinical trials $(24,31)$.

\section{Effect of CMAs on Global Metabolism}

We identified the significantly $($ FDR $<0.05)$ different plasma metabolites on Day 84 vs Day 0 and found that the plasma levels of 132 metabolites were significantly different in the CMA group (Figure 5, Dataset S5). Evaluation of plasma metabolites that differed significantly on Day 84 vs Day 0 in each group showed that a larger number of metabolites related to amino acid $(n=53)$, lipid metabolism $(n=42)$ and other metabolic pathways $(n=37)$ were altered in the CMA group compared to the placebo group (Figure 5, Dataset S5). 
medRxiv preprint doi: https://doi.org/10.1101/2021.07.14.21260511; this version posted August 15, 2021. The copyright holder for this preprint

$\mathrm{N}$-acetyl aspartate (NAA) is one of the most abundant brain metabolites and its reduced plasma levels are associated with brain tissue damage. Previous research revealed the importance of NAA to maintain energy metabolism in the central nervous system (32). In our study, we observed that plasma levels of NAA significantly increased on Day 84 vs Day 0 in the CMA group (Figure 5B, Dataset S5). Another upregulated metabolite on Day 84 vs Day 0 in the CMA group is sarcosine (a derivative of glycine) which has been widely studied for its improving effects of cognitive symptoms by different pharmacological activities in neurons (33). Of note, quinolinic acid (an endogenous excitotoxin acting on N-Methyl-D-aspartate receptors leading to neurotoxic damage) levels significantly decreased on Day 84 vs Day 0 only in the CMA group (Figure 5B, Dataset S5).

Increased plasma homocysteine levels is a known risk factor for $\mathrm{AD}$, and several animal studies implicated the promising results of methionine restriction $(34,35)$. In our clinical trial, plasma levels of S-adenosylhomocysteine as well as 2,3-dihydroxy-5-methylthio-4pentenoate (DMTPA) and N-acetyl taurine were significantly downregulated on Day 84 vs Day 0 in the CMA group (Figure 5B, Dataset S5). Of note, reductions of these metabolites are significantly correlated with serine and NR supplementation (Figure 6A, Dataset S6).

Increased plasma levels of metabolites in the kynurenine pathway are associated with $\mathrm{AD}$ severity (34). In our study, we found that plasma levels of kynurenate and 8methoxykynurenate were significantly lower on Day 84 vs Day 0 in the CMA group (Figure 5B, Dataset S5). Reduction in the plasma level of kynurenate was positively correlated with plasma serine levels (Dataset S6). Kynurenate, which has a prooxidant effect, is the product of the tryptophan degradation pathway. Its aerobic irradiation produces superoxide radicals and leads to cytochrome $\mathrm{C}$ reduction (36). It has been reported that increased levels of kynurenine lead to cell death through the reactive oxygen species (ROS) pathway in nature killer (NK) cells (37) and lower blood pressure in systemic inflammation (38).

Emerging evidence indicates a link between the abnormal kidney function and AD, but the potential impact of kidney on cognitive impairment is still undetermined (39). Recent studies showed that plasma levels of N,N,N-trimethyl-5-aminovalerate are involved in lysine metabolism, and serve as an indicator of elevated urinary albumin excretion (40). Here, we found that the plasma level of N,N,N-trimethyl-5-aminovalerate was significantly decreased on Day 84 vs Day 0 in the CMA group (Figure 5B, Dataset S5) and significantly inversely 
medRxiv preprint doi: https://doi.org/10.1101/2021.07.14.21260511; this version posted August 15, 2021. The copyright holder for this preprint

correlated with the plasma level of serine and NR. Moreover, the plasma level of creatinine was also significantly decreased on Day 84 vs Day 0 in the CMA group (Figure 5B, Dataset S5). The plasma reduction on creatinine is inversely correlated with the plasma level of serine (Dataset S6). Additionally, our analysis revealed decreased levels of several metabolites belonging to histidine metabolism in the CMA group on Day 84 vs Day 0. Among those Nacetyl-1-methylhistidine is associated with chronic kidney disease and showed a significant negative correlation with serine supplementation (Figure 5B, Dataset S6). Also, we found that plasma levels of metabolites related to the urea cycle (3-amino-2-piperidone, arginine, homoarginine, N-alpha-acetylornithine, ornithine and pro-hydroxy-pro) were significantly decreased in the CMA group on Day 84 vs Day 0 (Figure 5B, Dataset S5) and inversely correlated with the plasma level of serine and NR (Dataset S6).

Lipids play a fundamental role in the pathophysiology of neurodegenerative diseases, including AD. Specific lipid species of cellular membranes (e.g., cholesterol and sphingolipids) are not only structural components of cell membranes but also regulate a plethora of critical aspects of brain functions(41). In our study, plasma levels of a considerable number of metabolites associated with sphingomyelins and fatty acid metabolism (acyl carnitines) were significantly increased on Day 84 vs Day 0 in the CMA group (Figure 5C, Dataset S5). Interestingly, plasma levels of pregnenolone steroids and 2R,3R-dihydroxybutyrate were significantly decreased on Day 84 vs Day 0 (Figure 5C, Dataset S5). These alterations were significantly positively correlated with carnitine and serine levels (Dataset S6).

\section{Effect of CMAs on Plasma Proteins}

Plasma levels of 1466 protein markers were measured with the plasma proteome profiling platform Proximity Extension Assay quantifying the plasma level of target proteins. After quality control and exclusion of proteins with missing values in more than $50 \%$ of samples, 1463 proteins were analysed (Dataset S7). Proteins whose levels differed significantly between the visits in the CMA and placebo groups are listed in Dataset S7.

We analysed the effect of CMAs on plasma protein profile and found that 22 proteins were significantly (p-value<0.01) different in the CMA group on Day 84 vs Day 0. Nineteen of these proteins were significantly decreased, whereas 3 of these proteins were significantly increased on Day 84 vs Day 0. After filtering out the proteins based on $\log 2 \mathrm{FC}$, we found that 
medRxiv preprint doi: https://doi.org/10.1101/2021.07.14.21260511; this version posted August 15, 2021. The copyright holder for this preprint

the plasma levels of PSPN, OSM, PADI4, PDGFC, SCGN, LTBP3, CLEC4G, MERTK, WNT9A, ISM1, ASAH2, CES3, HPGDS, NPY, THPO, SIGLEC6, GDNF, PADI2 and EGFL7 were significantly downregulated in the CMA group. The plasma level of KLB, BGN, and ST3GAL1 was significantly upregulated in the CMA group (Figure 6B, Dataset S7). We observed that only 1 significantly ( $\mathrm{p}$-value $<0.01$ ) altered protein - EGFL7 upregulated- in the placebo group (Figure 6B, Dataset S7). Hence, we observed that plasma levels of proteins are significantly altered in response to CMA treatment.

The proteomic analysis in this study revealed significant alteration in levels of several critical proteins that play an essential role in the pathogenesis of AD. For instance, levels of MertK (42, 43), EGFR (44, 45), oncostatin (46-50), PAD4 (51, 52), LTGF (53-57), and TPO (58), known as a strong inducer of neuro-inflammation, amyloid production and apoptosis, decreased. In contrast, proteins with neuroprotective and pro-cognitive properties, such as Klotho $(59,60)$ and ST3GAL1 (61), increased after CMA treatment. More interestingly, the majority of the analysed proteins were also found to be significantly altered in recent human $\mathrm{AD}$ studies $(53-57,62-68)$. KlothoB levels were also significantly altered after CMA treatment, consistent with their neuroprotective role as a cofactor and neurotrophic factor. In this context, recent studies have shown that KlothoB indirectly regulates glucose and energy metabolism through F2F1, expressed in certain areas of the brain involved in learning and memory (67). Moreover, GABA signalling has also been shown to play a critical role in mediating the detrimental effects of increased dihydroxybutyrate levels in the progression of MCI (69). Interestingly, our metabolomic study indicated decreased post-therapeutic dihydroxybutyrate levels. Although the exact pathways involved in the metabolic generation of DHBA are still far from clear, it has been hypothesized that dihydroxybutyrate levels may be a compensatory response to increased cellular stress secondary to compromise of the Krebs cycle function, creating an alternative energy production pathway in AD (69). This represents indirect evidence to suggest that our treatment exhibits an energetic regulatory function.

\section{Integrative Multi-Omics Analysis}

Multi-omics data integrations have been proven to give novel insights and a more holistic view of the human body, in both healthy and disease states (70). In this study, we generated an integrative multi-omics network using metabolomics and proteomics data, coupled with detailed clinical variables, to understand the functional relationships between analytes from the same and different omics data types. We generated the network using the method used in 
medRxiv preprint doi: https://doi.org/10.1101/2021.07.14.21260511; this version posted August 15, 2021. The copyright holder for this preprint

iNetModels (71), to which we also deposited our network. The network consists of 937,282 edges from 2,273 nodes (36.3\% network density, Dataset S8).

We extracted a sub-network to highlight the interactions between the individual metabolic activators, cognitive function (ADAS-Cog scores), two highlighted proteins (OSM and PSPN), and their top neighbours (Figure 6C). From the sub-network, ADAS-Cog was shown to be negatively associated with carnitine (and its derivatives) and nicotinamide associated metabolites, where the metabolic cofactors were negatively associated with fatty acid and histidine metabolism. Finally, we observed that, among others, OSM and PSPN were positively associated with immune and cell cycle-related proteins.

Subsequently, we performed centrality analysis to identify the most central analytes in the networks. The top 20 most central metabolites were dominated by amino acid metabolites (tryptophan, glutamate, and branched-chain amino acid metabolism) and lipid metabolites (androgenic steroid pathway), where top proteins were related to, among others, short- and long-term memory (CALB1), lipid metabolism (PLA2G10), and immune response (SELPLG, CLEC4D, and LGALS7).

Furthermore, we performed community analysis within the network using the Leiden algorithm. We discovered 3 modules that showed significant interaction among the members. In cluster-0, the biggest clusters, the top nodes were related to tryptophan metabolism (indoleacetate), fatty acid metabolism (3-hydroxyoctanoate), and steroid metabolism (11ketoetiocholanolone glucuronide and 11-beta-hydroxyetiocholanolone glucuronide). Moreover, we found 2 top proteins in the same cluster, ACTA2 and IGFBP1, that have been associated with $\mathrm{AD}(72,73)$. In cluster-1, the top nodes were associated with leucine metabolism (3-hydroxy-2-ethylpropionate), ceramide phosphatidylethanolamine, and a carnitine metabolite (erucoylcarnitine), meanwhile, cluster-2's central nodes were related to methionine metabolism and aminosugar metabolism ( $\mathrm{N}$-acetylglucosamine/Nacetylgalactosamine). These results showed that the integrative multi-omics network analysis can be used to strengthen the results from single omics analyses and identify key analytes associated with AD. Moreover, it provided new insights by elucidating the functional relationships within and between different omics data.

In evaluating the correlations between each cofactor (used in the present study for therapeutic purposes) and clinical, metabolic, and proteomic parameters, we identified significant 
medRxiv preprint doi: https://doi.org/10.1101/2021.07.14.21260511; this version posted August 15, 2021. The copyright holder for this preprint

correlations between serine, carnitine, cysteine, and nicotinamide levels and improved peripheral blood parameters, such as liver function, $\mathrm{CBC}$, and glycated hemoglobin (HbA1c), which are relevant to the pathogenesis of AD. Accordingly, improved ADAS-Cog scores were also associated with changes in serum serine and carnitine, which fit well with their well-known pro-cognitive and energy-boosting effects. Similar results were also observed for metabolomic and proteomic data. The majority of the cofactors exhibited significant correlations with improved metabolites and proteins (either increased or decreased) relative to a slower degeneration process in $\mathrm{AD}$. It is worth mentioning here that that two of the proteins, OSM and PSPN, most strongly associated with other beneficial protein metabolites, were also related to several critical amino acid alterations, such as spermidine and hypotaurine, which may suggest a metabolic shift from the protein to amino acid metabolism to compensate the energy deficit reported in AD.

\section{DISCUSSION}

Here, we show that oral administration of CMAs has profound effect on cognitive function after only 84 days of treatment in AD patients based on ADAS-Cog scores. We showed that cognitive functions in AD patients is improved 29\% in the CMA group whereas $14 \%$ in the placebo group after 84 days of CMAs administration. We also recently tested the effect of CMAs administration on an independent cohort of Parkinson's disease patients in a Phase 2 clinical trial, and found that oral CMAs administration has a profound effect on cognitive function without altering motor scores in Parkinson's Disease (74). As the increased Montreal Cognitive Assessment (MoCA) scores is known as the indicator of the increased cognitive functions in PD patients, we observed that the mean MoCA scores were significantly higher in the CMA group both on Day 28 vs Day 0 (log2FoldChange $(\mathrm{FC})=0.17, \quad(13 \%$ improvement), $\mathrm{p}=0.001)$ and on Day 84 vs Day $0(\log 2 \mathrm{FC}=0.27,(21 \%$ improvement $), \mathrm{p}=$ 0.0001). We also observed a significant increase on MoCA scores in placebo group on Day 28 vs Day $0 \quad(\log 2 \mathrm{FC}=0.16$, (12\% improvement), $\mathrm{p}=0.001)$ and on Day 84 vs day 0 $(\log 2 \mathrm{FC}=0.15,(11 \%$ improvement $), \mathrm{p}=0.04)$ due to the recommendations of exercise and Mediterranean diet to all PD patients participated in the trial. Notably, the degree of increase of MoCA was much higher on Day 84 vs Day 0 in the CMA group than in the placebo group, suggesting the PD patients benefitted from CMA treatment after 84 days of treatment. Even though, Montreal Cognitive Assessment (MoCA) scores have been used to evaluate the cognitive function in the PD patients, the effect of CMAs administration on cognitive function have been verified in an independent patient group with different neurological diseases. 
medRxiv preprint doi: https://doi.org/10.1101/2021.07.14.21260511; this version posted August 15, 2021. The copyright holder for this preprint

Clinically, when AD patients were stratified by high and low ADAS-Cog scorer, we observed that patients with lower ADAS-Cog scores in the placebo group also showed improved cognitive function similar to the CMA group. However, for the patients with higher ADASCog scores, cognitive function was not improved in the placebo group, while a positive effect was observed for the patients treated with metabolic activators. This is particularly interesting, since this patient group lacks current therapeutic regimes, except for palliative support. Apart from clinical severity, we also observed that various clinical variables were also related to the treatment response. For example, patients with low ALT, who did not drink alcohol or smoke, and who had signs of an increased metabolic load (i.e., increased HbA1c and insulin levels) or impaired $\mathrm{CBC}$ values responded better to treatment. Additionally, the treatment significantly improved the altered metabolic and $\mathrm{CBC}$ parameters described above.

The effect of oral administration of CMAs was substantiated with a comprehensive analysis of protein and metabolites in the plasma of the patients using a multi-omics analytical platform. The clinical results are consistent with the genome-scale metabolic modelling of more than $600 \mathrm{AD}$ patients showing evidence of mitochondrial dysfunction. It is also consistent with the results from an animal model demonstrating improved AD-associated histological parameters in animals treated with an oral administration of CMAs. Thus, the present study suggests an attractive therapeutic regime for improving mitochondrial dysfunction in $\mathrm{AD}$ patients.

The metabolomics data confirmed the expected biological outcomes of CMA treatment. Levels of plasma nicotinamide and related metabolites increased, suggesting that NR provided sufficient substrate for mitochondrial fatty acid oxidation. In addition to its role as a cellular metabolite, NAD+ functions as an essential cofactor for the DNA repair protein PARP1 (17). Hyperactivation of PARP1 and decreased NAD+ have been already identified in the brains of patients with $\mathrm{AD}(75,76)$. Serine plasma levels also increased, suggesting that CMA treatment improves the serine deficiency associated with AD. For instance, a recent study showed that the adenosine triphosphate (ATP)-reducing the effect of glucose hypometabolism was restored with oral serine supplementation, suggesting the potential use of oral serine as a ready-to-use therapy for AD (77). The exact mechanism of action also applies to cysteine. As a glutathione precursor, cysteine is even more specific in acting as an antioxidant and anti-inflammatory agent, maintaining the mitochondrial energetic homeostasis 
medRxiv preprint doi: https://doi.org/10.1101/2021.07.14.21260511; this version posted August 15, 2021. The copyright holder for this preprint

and key neurotransmitter systems, such as glutamate, involved in learning and memory (78, 79). Accordingly, NAC has been tested as a medication in $\mathrm{AD}$ and found to exhibit effects suggestive of future potential use as an alternative medication (80). More importantly, fatty acid oxidation and carnitine metabolism were significantly facilitated, as shown by the robust increase in plasma levels of carnitine. These findings fit well with recent human data showing that severe disturbances in carnitine metabolism frequently occur in individuals with $A D$, in association with severe mitochondrial dysfunction $(81,82)$. Cristofano et al. showed a progressive decrease in carnitine serum levels in individuals shifting from normal status to $\mathrm{AD}$, suggesting that decreased serum concentrations of carnitine may predispose to AD (83). In support of this hypothesis, human clinical studies have demonstrated the pro-cognitive effects of carnitine in mild cognitive impairment and $\mathrm{AD}(84-86)$. This, in turn, led to the suggestion that stabilizing the bioenergetic balance may slow or even reverse mild cognitive impairment and the progression of dementia in patients with AD.

In addition, the levels of tryptophan metabolites, including kynurenate, kynurenine, and tryptophan betaine, decreased significantly after CMA treatment. Increased levels of these metabolites were previously shown to be associated with increased neurodegeneration and clinical cognitive impairment through an increased oxidative load and the formation of neurofibrillary tangles (NFTs) $(87,88)$. For instance, recent data showed a synergistic relationship between $\beta$-amyloid 1-42 and enzymatic activations of the tryptophan kynurenine pathway, resulting in increased oxidative stress, which may be associated with the formation of NFTs and senile plaque development (89). Also, one recent study revealed that tryptophan2,3-dioxygenase (TDO) was highly expressed in the brains of patients with AD and colocalized with quinolinic acid, NFTs, and amyloid deposits in the hippocampus of postmortem brains of patients with $\mathrm{AD}(90)$.

We also observed significantly increased levels of NAA, sarcosine, methionine, cysteine, and S-adenosylmethionine (SAM) and decreased levels of histidine, tryptophan quinolate, and urea cycle metabolites, which play a critical role in cognitive and mitochondrial functions. For instance, increased NAA may provide an additional energy source for intercellular metabolite trafficking during the neurodegenerative process, especially when glucose metabolism is downregulated (32). Similarly, increased sarcosine levels may boost cognition, as previously shown in patients with schizophrenia, in which oxidative damage and impaired glucose metabolism play key roles (91). In addition, decreased histidine metabolism and other 
medRxiv preprint doi: https://doi.org/10.1101/2021.07.14.21260511; this version posted August 15, 2021. The copyright holder for this preprint

decreased markers, such as homocysteine and S-adenosylhomocysteine (SAH) found in our treatment group, have been already shown to slow the cognitive ageing process appropriately downregulated (92). For instance, increased plasma homocysteine levels are a known risk factor for $\mathrm{AD}$, whereas a low leucine and arginine diet yield beneficial cognitive effects (93).

Unexpectedly, CMAs rapidly lowered uric acid and associated metabolites levels. Uric acid stimulates inflammation either directly or by activating NLRP3 inflammasomes (94). Although the extent to which uric acid reduction contributed to the regression in cognitive impairment is unclear, it likely that it is linked to the improvement in the metabolic homeostasis. A good example is a recent clinical study showing increased urea metabolism in patients with AD (95). Accordingly, decreased taurine levels and urea metabolites are associated with a diminished risk of dementia (96). The majority of clinical study findings collectively agree with our results, showing significantly dysregulated baseline metabolites, which normalized with treatment.

Of note, to date, a few studies aimed to identify global changes in metabolites and metabolic pathways in $\operatorname{AD}(15,97,98)$. Among these, some studies highlighting that lipid dysfunction also plays an important role in the pathophysiology of AD (99). In terms of lipid metabolism, significant differences in the levels of some compounds have been observed in patients with AD. Despite some discrepant trends in cross-sectional studies examining the levels of lipids in AD patients $(100,101)$, the plasma levels of sphingolipids, sphingomyelins (102, 103), acylcarnitines (104)and phosphatidylcholines(PC) (105-107) exhibited statistically lower concentrations in patients with $\mathrm{AD}$, even in the preclinical stages of the disease (18). In addition, a significant correlation among different lipid metabolites, tau and amyloid pathology, brain atrophy and cognitive decline was observed in a AD human study (18). An autopsy study of frontal cortex metabolites from patients with AD showed that impaired glycerophospholipid metabolism was involved in six central metabolic pathways reported to be altered in the disease (108). In brief, we observed significantly increased post-therapeutic levels of lipid metabolites, previously reported to decrease in patients with $\mathrm{AD}$, including sphingomyelin, carnitine and carnitine-related by-products.

Despite insufficient clinical AD data concerning cholesterol metabolites and dicarboxylic acids (DCAs), we observed significantly lower levels of these metabolites after CMA treatment (109). Levels of pregnanediol, a metabolite of pregnenolone, and DCAs, end- 
medRxiv preprint doi: https://doi.org/10.1101/2021.07.14.21260511; this version posted August 15, 2021. The copyright holder for this preprint

products of $\beta$ - or omega oxidation, which were observed as decreased in the present study, were previously reported to be lower in the urine of patients with $\mathrm{AD}(110,111)$. Considering the neurotoxic role of bile acids, along with the oxidative properties of DCAs, the detection of decreased levels of bile acid metabolites and DCA products in the present study is therefore not surprising. Similarly, allopregnanolone has already been reported to result in deleterious effects on cognitive functions through gamma-aminobutyric acid (GABA) signalling (112). Also, increased bile acid levels have been reported in mild cognitive impairment (MCI) and AD. In contrast, bile acids strongly inhibited the cysteine catabolic pathway in the preclinical period, resulting in depletion of the free cysteine pool and reduction of antioxidant glutathione concentrations(113).

Although there has been no direct evidence relating plasma ascorbic acid (AA) levels to the pathogenesis of $\mathrm{AD}$, our finding of decreased plasma levels after CMAs may be related to increased brain concentrations and central consumption of ascorbic acid in cognitively improved patients with $\mathrm{AD}$. In this respect, and contrast to other metabolites, a direct transmission from the periphery to the brain suggested that AA might play a 'nourishing' role in the brain. A direct correlation between brain levels of AA and dementia was eventually confirmed by a recent study showing that a high CSF: plasma AA ratio was a marker of a "healthier" brain, better able to cope with the neurodegenerative process in AD (114).

However, despite these promising human data, plasma levels of several proteins in AD observed after CMA treatment in the present study were inconsistent with their established beneficial role under experimental conditions. For example, PSP, which decreased in the present study, is a novel neurotrophic factor exhibiting significant similarities to GDNF by exerting intense neurotrophic activity, specifically on central neurons $(115,116)$. Such discrepancies are not easily explained, even if the different results obtained in peripheral blood can be correlated with improved clinical status. A finding of decreased or increased post-therapeutic levels of these proteins in patients with AD may reflect a treatment-related alteration in their cellular processing in the central nervous system, resulting in variability in protein production and/or degradation in peripheral blood. In addition, controversial data have been reported regarding the concentrations of most of these proteins in patients with AD (102, 117-120). Also, due to the high heterogeneity of clinical cohorts and the restricted number of patients, the reproducibility of previous proteomics studies' results is noticeably low. 
medRxiv preprint doi: https://doi.org/10.1101/2021.07.14.21260511; this version posted August 15, 2021. The copyright holder for this preprint

Our therapeutic data fits well with recent ROSMAP transcriptomic data in AD patients, showing impaired glycolysis, urea cycle, and fatty acid metabolism that was considerably normalized after the CMA treatment. For instance, our transcriptomic analysis showed increased expressions of pyruvate dehydrogenase kinase 4 (PDK4), carnitine palmitoyltransferase (CPT1A) and hexokinase 2 (HK2). It decreased expressions of acyl-CoA dehydrogenases (ACADs), ATP synthase (ATP5MGL), and acetyl- and acyltransferases (GCNT7, MBOAT4, GALNT17) which have been already reported to link/associate with critical energetic deficiencies due to decreased glycolysis and lipid oxidation metabolism. Consistent with the above-mentioned expression levels, we observed increased metabolites in the cytosol, including carnitine, spermine, serine, alanine, glucose 6-phosphate, and acyl-CoA responsible for glycolytic and lipid metabolism-related energetic cascades. Also, our animal data confirmed the beneficial effects of our CMAs, showing the most substantial impacts on hyperemia, neuronal degeneration and necrosis in HFD animals.

A few limitations of the study need to be considered. First, the treatment effect was assessed by clinical evaluation and omics-analysis. Thus, our findings warrant a clinical trial with neuroimaging analysis to delineate the impact of CMAs on functional and structural brain alterations. Second, the link between systemic and CNS alterations and their relations to the AD pathology, i.e., amyloid and tau aggregation, has not been evaluated. Thus, further amyloid or tau-based neuroimaging combined with CSF evaluation should be pursued.

In summary, the human phase 2 clinical study supports the data from animal models and genome-scale metabolic modelling suggesting that oral administration of metabolic activators can improve the mitochondrial dysfunction in AD patients. The safety profile of metabolic activators in these patients was consistent with the results of our previous one-day calibration study and clinical trials, including only a single component of the CMAs (20). Our present study showed that CMAs was safe and well-tolerated in patients with $\mathrm{AD}$, and no major safety concerns were identified. Importantly, CMAs improved cognition and serum markers in these patients after only 12 weeks of treatment. These findings suggest that targeting multiple pathways by metabolic activators is a potentially effective therapeutic strategy for $\mathrm{AD}$. 
medRxiv preprint doi: https://doi.org/10.1101/2021.07.14.21260511; this version posted August 15, 2021. The copyright holder for this preprint

\section{MATERIAL AND METHODS}

\section{Transcriptomics data analysis of the brain in AD patients}

The mRNA expression profiles of $629 \mathrm{AD}$ and 704 control samples were obtained from the Religious Orders Study and Rush Memory Aging Project (ROSMAP) datasets (26-28). The data has been normalized by quantile scaling, TMM normalization, Pareto scaling, and then limma removeBatchEffect (121). DESeq2 (122) was used to identify the differentially expressed genes (DEGs) with uniform size factors. Ensembl Biomart (123) was used to mapping different gene accession IDs or symbols. Gene set enrichment (GSE) was performed by using piano (124). The top 5\% DEGs by DESeq2 statistic were accepted for GSE analysis. To identify the significantly changed metabolites in $\mathrm{AD}$, we performed the Reporter metabolite analysis based on the RAVEN Toolbox 2.0 reporterMetabolites function (125), which uses DEGs information through the network topology of the reference metabolic model. The iBrain2845 (29) genome-scale metabolic model was used as the reference metabolic model. KEGG Pathway Mapper (126) was used to identify predicted changed pathways based on maps M01200, M01212, and M01230

\section{Animal study design}

12-week old female Sprague-Dawley rats (weighing 320-380g) were kept at a controlled temperature of $22 \pm 2{ }^{\circ} \mathrm{C}$ and a controlled humidity of $50 \pm 5 \%$ on a 12-hour light/dark cycle. Food and water were available ad libitum. The animal experiments are performed at Medical Experimental Research Center, Atatürk University, Erzurum, Turkey. All experiments for the treatment of the animals were approved by the Ethics Committee of Atatürk University, and were conducted following the National Institutes of Health Guide for Care and Use of Laboratory Animals.

After acclimation to laboratory conditions for 1 week, rats were randomly divided into two dietary regimens receiving either a Chow diet $(\mathrm{CHOW})$ or a high-fat diet (HFD. Group 1 $(n=4)$ were fed with only regular CD for 5 weeks; Group $2(n=4)$ were fed with CD and treated with STZ. The remaining animals in Group $3(n=4)$ were fed with HFD for 3 weeks and sacrificed to verify the model development. Groups 4-10 were fed with HFD for 5 weeks. After 3 weeks, the animals in Groups 5-10 were treated with STZ and administered with individual or combined metabolic activators, including L-serine, NAC, LCAT and NR for 2 weeks.

\section{Intracerebroventricular-streptozotocin (STZ) injection}

Before surgical procedures, the rats were anesthetized by intraperitoneal administration of ketamine-xylazine (50 mg/kg ketamine and $5 \mathrm{mg} / \mathrm{kg}$ xylazine) and placed individually in the 
medRxiv preprint doi: https://doi.org/10.1101/2021.07.14.21260511; this version posted August 15, 2021. The copyright holder for this preprint

stereotaxic instrument (Stoelting, Illinois, USA). Stereotaxic coordinates for injection were $0.8 \mathrm{~mm}$ posterior to the bregma, $1.5 \mathrm{~mm}$ lateral to the sagittal suture and $3.6 \mathrm{~mm}$ below the brain surface (127). Then, 10 microliters of STZ (3 mg/kg, Sigma-Aldrich, Darmstadt, Germany) were injected over 3 min with a Hamilton micro-syringe into the bilateral ventricle. The injection needles were left in place for an additional 2 min to allow diffusion.

\section{CMAs administration to animals}

For carrying out the treatment experiments, the HFD rats were randomly separated into 9 (Groups 5-10) subgroups ( $\square=\square 4$ ) and all treated with $3 \square \mathrm{mg} / \mathrm{kg} \mathrm{STZ} \mathrm{(10 \square \mu L,} \mathrm{icv).} \mathrm{Group}$ 5, treated only with STZ; Group 6, treated with STZ and serine $(1000 \mathrm{mg} / \mathrm{kg}$ once daily by oral gavage); Group 7, treated with STZ and NAC (300 mg/kg once daily by oral gavage); Group 8, treated with STZ and LCAT (100 mg/kg once daily by oral gavage); Group 9, treated with STZ and NR (120 mg/kg once daily by oral gavage); Group 10, treated with STZ and serine $(1000 \mathrm{mg} / \mathrm{kg}$ once daily by oral gavage), NAC (300 mg/kg once daily by oral gavage), LCAT (100 mg/kg/day) and NR (120 mg/kg once daily by oral gavage).

The body weight of the rats was recorded each week (Dataset S2). After 5 weeks, all animals were anesthetized with isoflurane and sacrificed. Blood samples were collected from the abdominal aorta and centrifuged at $8000 \square \mathrm{rpm}$ for $15 \square \mathrm{min}$ at $4 \square{ }^{\circ} \mathrm{C}$ for blood biochemistry analysis using automatic chemical analyser. The internal organs, including the heart, adipose tissues, liver, kidney, brain, muscle, intestine (duodenum, ileum, jejunum), pancreas, colon and stomach were immediately removed and then snap-frozen in liquid nitrogen and stored at $\square 80^{\circ} \mathrm{C}$.

Liver and brain tissue samples obtained due to the experimental procedure were fixed in $10 \%$ buffered formalin solution for 48 hours. Following the routine tissue procedure, the tissues were embedded in paraffin blocks and $4 \mu \mathrm{m}$ thick sections were taken from each block. Preparations prepared for histopathological examination were stained with hematoxylin-eosin (HE) and examined with a light microscope (Olympus BX51, Germany). According to histopathological findings sections were evaluated by independent pathologist and scored as absent (-), very mild (+), mild (++), moderate $(+++)$ and severe $(++++)$.

\section{Immunohistochemical examination}

Tissue sections taken on the adhesive (poly-L-Lysin) slides for immunoperoxidase examination were deparaffinized and dehydrated. After washing, the tissues with suppressed endogenous peroxidase activity in $3 \% \mathrm{H}_{2} \mathrm{O}_{2}$ were boiled in antigen retrieval solution. To prevent nonspecific background staining in the sections, protein block compatible with all primary and secondary antibodies was dropped and incubated for 5 minutes. Caspase 3 (cat 
medRxiv preprint doi: https://doi.org/10.1101/2021.07.14.21260511; this version posted August 15, 2021. The copyright holder for this preprint

no:sc-56053 dilution ratio:1/100 US) for liver tissues and 8-OH-dG (Cat no: sc-66036 dilution ratio: 1/100 US) for brain tissues was used as the primary antibody. 3-3' Diaminobenzidine (DAB) chromogen was used as chromogen, and according to their immunopositivity, sections were evaluated by independent pathologist and scored as absent (-), very mild (+), mild (++), moderate (+++) and severe (++++).

\section{Immunofluorescence examination}

Tissue sections taken on the adhesive (poly-L-Lysin) slides for immunoperoxidase examination were deparaffinized and dehydrated. After washing, the tissues with suppressed endogenous peroxidase activity in $3 \% \mathrm{H}_{2} \mathrm{O}_{2}$ were boiled in antigen retrieval solution. To prevent nonspecific background staining in the sections, protein block compatible with all primary and secondary antibodies was dropped and incubated for $5 \mathrm{~min}$. For liver tissues, primary antibody 8-OHdG (Cat No: sc-66036 Dilution Ratio:1/100 US) was dropped and incubated at $37^{\circ} \mathrm{C}$ for $1 \mathrm{~h}$. After washing, secondary FITC (Cat No: ab6717 Dilution Ratio:1/500 UK) was dropped and incubated at $37^{\circ} \mathrm{C}$ for $30 \mathrm{~min}$. The other primary antibody H2A.X (Cat No:I 0856-1 Dilution Ratio:1/100 US) was dropped and incubated at $37^{\circ} \mathrm{C}$ for 1 h. After washing, secondary Texas Red (Cat No: sc-3917 Dilution Ratio:1/100 US) was dropped and incubated at $37^{\circ} \mathrm{C}$ for 30 min. DAPI (Cat No:D-1306 Dilution Ratio:1/200 US) was dripped onto the washed tissues and incubated in the dark for $5 \mathrm{~min}$, then glycerine was sealed. For brain tissues, primary antibody Caspase 3 (Cat No:sc-56053 Dilution Ratio:1/100 US) was dropped and incubated for $1 \mathrm{~h}$ at $37^{\circ} \mathrm{C}$. After washing, secondary FITC (Cat No: ab6717 Dilution Ratio:1/500 UK) was dropped and incubated at $37^{\circ} \mathrm{C}$ for $30 \mathrm{~min}$. The other primary antibody H2A.X (Cat No: I 0856-1 Dilution Ratio:1/100 US) was dropped and incubated at $37^{\circ} \mathrm{C}$ for $1 \mathrm{~h}$. After washing, secondary Texas Red (Cat No: sc-3917 Dilution Ratio:1/100 US) was dropped and incubated at $37^{\circ} \mathrm{C}$ for $30 \mathrm{~min}$. DAPI (Cat No:D-1306 Dilution Ratio:1/200 US) was dripped onto the washed tissues and incubated in the dark for 5 min, then glycerine was sealed. Sections were examined under a fluorescence microscope (ZEISS Germany) by an independent pathologist and, according to their immunopositivity evaluated as absent (-), very mild (+), mild (++), moderate $(+++)$ and severe $(++++)$.

\section{Clinical Trial Design and Oversight}

Patients for this randomized, double-blinded, placebo-controlled, phase 2 study were recruited at the Faculty of Medicine, Alanya Alaaddin Keykubat University, Antalya, Turkey and Faculty of Medicine, Istanbul Medipol University, Istanbul, Turkey. Written informed consent was obtained from all participants before the initiation of any trial-related procedures. The safety of the participants and the risk-benefit analysis was overseen by an independent 
medRxiv preprint doi: https://doi.org/10.1101/2021.07.14.21260511; this version posted August 15, 2021. The copyright holder for this preprint

external data-monitoring committee. The trial was conducted following Good Clinical Practice guidelines and the principles of the Declaration of Helsinki. The ethics committee approved the study of Istanbul Medipol University, Istanbul, Turkey, and retrospectively registered at https://clinicaltrials.gov/ with Clinical Trial ID: NCT04044131.

\section{Participants}

Patients were enrolled in the trial if they were over 50 years of age with mild to moderate AD according to ADAS-cog (AD Assessment Scale-cognitive subscale; ADAS $\geq 12$ ) and the Clinical Dementia Rating Scale Sum of Boxes (CDR-SOB; CDR $\leq 2)$. Patients who had a history of stroke, severe brain trauma, toxic drug exposure were excluded. The main characteristics of the patients are summarized in Dataset S3. The inclusion, exclusion, and randomization criteria are described in detail in the Supplementary Appendix.

\section{Randomization, Interventions, and Follow-up}

Patients were randomly assigned to receive CMAs or placebo (2:1). Patient information (patient number, date of birth, initials) was entered into the web-based randomization system, and the randomization codes were entered into the electronic case report form. All clinical staff were blinded to treatment, as were the participants.

Treatment started on the day of diagnosis. Both placebo and CMAs were provided in powdered form in identical plastic bottles containing a single dose to be dissolved in water and taken orally one dose in the morning after breakfast and one dose in the evening after dinner. Each dose of CMAs contained $3.73 \mathrm{~g}$ L-carnitine tartrate, $2.55 \mathrm{~g} \mathrm{~N}$-acetylcysteine, $1 \mathrm{~g}$ nicotinamide riboside chloride, and $12.35 \mathrm{~g}$ serine. All patients received one dose during the first 28 days and received two doses until Day 84. All patients came for a follow-up visit on Day 84. Further information is provided in the Supplementary Appendix.

\section{Outcomes}

The primary endpoint in the original protocol was to assess the clinical efficacy of CMAs in $\mathrm{AD}$ patients. For the primary purpose, the clinical differences in cognition of subjects receiving twelve-week treatment either with metabolic activators supplementation or placebo were determined. The primary analysis was on the difference in cognitive and daily living activity scores between the placebo and the treatment arms, which were assessed by MiniMental State Examination (MMSE), AD Assessment Scale-cognitive subscale (ADAS-Cog) and AD Cooperative Study - Activities of Daily Living (ADCS-ADL) in AD patients. The secondary aim in this study was to evaluate the safety and tolerability of CMAs. All protocol amendments were authorized and approved by the sponsor, the institutional review board or independent ethics committee, and the pertinent regulatory authorities. 
medRxiv preprint doi: https://doi.org/10.1101/2021.07.14.21260511; this version posted August 15, 2021. The copyright holder for this preprint

Number and characteristics of adverse events, serious adverse events, and treatment discontinuation due to CMAs were reported from the beginning of the study to the end of the follow-up period as key safety endpoints. The changes in vital signs baseline values, and the status of treatment were recorded at Day 0 and 84 . A complete list of the end points is provided in the Supplementary Appendix.

\section{Proteomics Analysis}

Plasma levels of proteins were determined with the Olink panel (Olink Bioscience, Uppsala, Sweden). Briefly each sample was incubated with DNA-labelled antibody pairs (proximity probes). When an antibody pair binds to its corresponding antigens, the corresponding DNA tails form an amplicon by proximity extension, which can be quantified by high-throughput, real-time PCR. Probe solution ( $3 \mu \mathrm{l})$ was mixed with $1 \mu \mathrm{l}$ of sample and incubated overnight at $4{ }^{\circ} \mathrm{C}$. Then $96 \mu \mathrm{l}$ of extension solution containing extension enzyme and PCR reagents for the pre-amplification step was added, and the extension products were mixed with detection reagents and primers and loaded on the chip for qPCR analysis with the BioMark HD System (Fluidigm Corporation, South San Francisco, CA). To minimize inter- and intrarun variation, the data were normalized to both an internal control and an interplate control. Normalized data were expressed in arbitrary units (Normalized Protein eXpression, NPX) on a $\log 2$ scale and linearized with the formula 2NPX. A high NPX indicates a high protein concentration. The limit of detection, determined for each of the assays, was defined as three standard deviations above the negative control (background).

\section{Untargeted Metabolomics Analysis}

Plasma samples were collected on Days 0 and 84 for nontargeted metabolite profiling by Metabolon (Durham, NC). The samples were prepared with an automated system (MicroLab STAR, Hamilton Company, Reno, NV). For quality control purposes, a recovery standard was added before the first step of the extraction. To remove protein and dissociated small molecules bound to protein or trapped in the precipitated protein matrix, and to recover chemically diverse metabolites, proteins were precipitated with methanol under vigorous shaking for 2 min (Glen Mills GenoGrinder 2000) and centrifuged. The resulting extract was divided into four fractions: one each for analysis by ultraperformance liquid chromatographytandem mass spectroscopy (UPLC-MS/MS) with positive ion-mode electrospray ionization, UPLC-MS/MS with negative ion-mode electrospray ionization, and gas chromatographymass spectrometry; one fraction was reserved as a backup.

\section{Determination of clinical variables informing response to CMAs administration}


The patient groups with low and high levels of each clinical parameter were established based on the median score for that clinical parameter across all patients on Day 0. Patients scoring at or below the median were placed in the low group; patients scoring above the median were placed in the high group. ADAS-Cog scores were measured over different time points and statistical significance was tested between time points by using a paired t-test. Clinical parameters were deemed informative for the response to CMAs if exactly one group (low or high) exhibited more statistically significant changes in ADAS-Cog in the CMA group than in the placebo group.

\section{Statistical Analysis}

Paired t-test was used to identify the differences in clinical parameters between time points and one-way ANOVA was used to find the shifts between CMA and placebo groups at each time point. For analysis of plasma metabolomics, we removed the metabolite profiles with more than $50 \%$ missing values across all samples. Metabolite changes between time points were analysed by paired t-test. Metabolite changes between CMA and placebo groups were analysed by one-way ANOVA. Missing values were removed in pairwise comparison. The pvalues were adjusted by Benjamini \& Hochberg method. Metabolites with a false-discovery rate of $5 \%$ were considered statistically significant. Two-sided Student's t-test was used for statistical analyses of plasma parameters in animal model, statistical significance was considered $\mathrm{p}<0.05$.

For analysis of plasma proteomics, we removed the protein profiles with more than $50 \%$ missing values across all samples. Pair t-test was used to identify the changes between time points and one-way ANOVA was used to identify the changes between different groups. $\mathrm{p}<0.01$ was considered statistically significant. Spearman correlation analysis was used to analyse the association between CMAs and clinical parameters or metabolomics or proteomics.

\section{Generation of Multi-Omics Network}

Multi-omics network was generated based on the Spearman correlations and the significant associations (FDR < 0.05) are presented. The analyses were performed with SciPy package in Python 3.7. Centrality analysis on the network was performed using iGraph Python.

\section{ACKNOWLEDGMENTS}


This work was financially supported by ScandiBio Therapeutics and Knut and Alice Wallenberg Foundation. The authors would like to thank the Metabolon Inc. (Durham, USA) for generation of metabolomics data, and ChromaDex Inc. (Irvine, CA, USA) for providing NR. AM and HY acknowledge support from the PoLiMeR Innovative Training Network (Marie Skłodowska-Curie Grant Agreement No. 812616) which has received funding from the European Union's Horizon 2020 research and innovation programme.

\section{CONFLICT OF INTEREST}

AM, JB and MU are the founder and shareholders of ScandiBio Therapeutics. The other authors declare no competing interests.

\section{SUPPORTING INFORMATION}

Supporting Information includes 8 Supplementary Datasets. 


\section{REFERENCES}

1. L. Trujillo-Estrada et al., In vivo modification of Abeta plaque toxicity as a novel neuroprotective lithium-mediated therapy for Alzheimer's disease pathology. Acta Neuropathol Commun 1, 73 (2013).

2. A. Nunomura, G. Perry, RNA and Oxidative Stress in Alzheimer's Disease: Focus on microRNAs. Oxidative Medicine and Cellular Longevity 2020, 2638130 (2020).

3. S. Lam et al., A systems biology approach for studying neurodegenerative diseases. Drug Discov Today 25, 1146-1159 (2020).

4. J. G. Mielke et al., A biochemical and functional characterization of diet-induced brain insulin resistance. J Neurochem 93, 1568-1578 (2005).

5. S. E. Arnold et al., Brain insulin resistance in type 2 diabetes and Alzheimer disease: concepts and conundrums. Nat Rev Neurol 14, 168-181 (2018).

6. Y. Wei et al., Ribosylation triggering Alzheimer's disease-like Tau hyperphosphorylation via activation of CaMKII. Aging Cell 14, 754-763 (2015).

7. F. Masciopinto et al., Effects of long-term treatment with pioglitazone on cognition and glucose metabolism of PS1-KI, 3xTg-AD, and wild-type mice. Cell Death Dis 3, e448-e448 (2012).

8. L. R. Wong, P. Wong, P. C. Ho, Metabolic Profiling of Female Tg2576 Mouse Brains Provides Novel Evidence Supporting Intranasal Low-Dose Pioglitazone for Long-Term Treatment at an Early Stage of Alzheimer's Disease. Biomedicines 8, (2020).

9. S. Makin, The amyloid hypothesis on trial. Nature 559, S4-s7 (2018).

10. B. M. Kuehn, In Alzheimer Research, Glucose Metabolism Moves to Center Stage. JAMA 323, 297-299 (2020).

11. Z. Chen, C. Zhong, Decoding Alzheimer's disease from perturbed cerebral glucose metabolism: implications for diagnostic and therapeutic strategies. Prog Neurobiol 108, 2143 (2013).

12. C. L. Powell, A. R. Davidson, A. M. Brown, Universal Glia to Neurone Lactate Transfer in the Nervous System: Physiological Functions and Pathological Consequences. Biosensors (Basel) 10, (2020).

13. D. Drulis-Fajdasz, A. Gizak, T. Wójtowicz, J. R. Wiśniewski, D. Rakus, Aging-associated changes in hippocampal glycogen metabolism in mice. Evidence for and against astrocyte-to-neuron lactate shuttle. Glia 66, 1481-1495 (2018).

14. J. Cummings, G. Lee, A. Ritter, M. Sabbagh, K. Zhong, Alzheimer's disease drug development pipeline: 2020. Alzheimers Dement (N Y) 6, e12050 (2020).

15. X. Pan et al., Alzheimer's disease-like pathology has transient effects on the brain and blood metabolome. Neurobiol Aging 38, 151-163 (2016).

16. Y. Dong, G. J. Brewer, Global Metabolic Shifts in Age and Alzheimer's Disease Mouse Brains Pivot at NAD+/NADH Redox Sites. J Alzheimers Dis 71, 119-140 (2019).

17. Y. Hou et al., NAD+ supplementation normalizes key Alzheimer's features and DNA damage responses in a new AD mouse model with introduced DNA repair deficiency. Proceedings of the National Academy of Sciences 115, E1876-E1885 (2018).

18. J. B. Toledo et al., Metabolic network failures in Alzheimer's disease: A biochemical road map. Alzheimers Dement 13, 965-984 (2017).

19. G. M. Sancesario, S. Bernardini, Alzheimer's disease in the omics era. Clin Biochem 59, 9-16 (2018).

20. C. Zhang et al., The acute effect of metabolic cofactor supplementation: a potential therapeutic strategy against non-alcoholic fatty liver disease. Mol Syst Biol 16, e9495 (2020).

21. A. Mardinoglu et al., The Potential Use of Metabolic Cofactors in Treatment of NAFLD. Nutrients 11, 1578 (2019).

22. A. Mardinoglu et al., Genome-scale metabolic modelling of hepatocytes reveals serine deficiency in patients with non-alcoholic fatty liver disease. Nat Commun 5, 3083 (2014). 
medRxiv preprint doi: https://doi.org/10.1101/2021.07.14.21260511; this version posted August 15, 2021. The copyright holder for this preprint (which was not certified by peer review) is the author/funder, who has granted medRxiv a license to display the preprint in perpetuity.

It is made available under a CC-BY-NC-ND 4.0 International license .

23. A. Mardinoglu, J. Boren, U. Smith, M. Uhlen, J. Nielsen, Systems biology in hepatology: approaches and applications. Nature Reviews Gastroenterology \& Hepatology 15, 365-377 (2018).

24. O. Altay et al., Combined Metabolic Activators Accelerates Recovery in Mild-to-Moderate COVID-19. Advanced Science n/a, 2101222 (2021).

25. A. Mardinoglu et al., An Integrated Understanding of the Rapid Metabolic Benefits of a Carbohydrate-Restricted Diet on Hepatic Steatosis in Humans. Cell Metab 27, 559-571 e555 (2018).

26. A. J. Myers et al., A survey of genetic human cortical gene expression. Nature Genetics 39, 1494-1499 (2007).

27. J. A. Webster et al., Genetic control of human brain transcript expression in Alzheimer disease. Am J Hum Genet 84, 445-458 (2009).

28. S. Mostafavi et al., A molecular network of the aging human brain provides insights into the pathology and cognitive decline of Alzheimer's disease. Nat Neurosci 21, 811-819 (2018).

29. S. Lam et al., Systems analysis reveals ageing-related perturbations in retinoids and sex hormones in Alzheimer's and Parkinson's diseases. bioRxiv, 2021.2006.2010.447367 (2021).

30. A. Mardinoglu et al., Integration of clinical data with a genome-scale metabolic model of the human adipocyte. Molecular systems biology 9, 649-649 (2013).

31. M. Zeybel et al., Combined Metabolic Activators Reduces Liver Fat in Nonalcoholic Fatty Liver Disease Patients. medRxiv, 2021.2005.2020.21257480 (2021).

32. J. R. Moffett, P. Arun, P. S. Ariyannur, A. M. A. Namboodiri, N-Acetylaspartate reductions in brain injury: impact on post-injury neuroenergetics, lipid synthesis, and protein acetylation. Front Neuroenergetics 5, 11-11 (2013).

33. S. P. Singh, V. Singh, Meta-analysis of the efficacy of adjunctive NMDA receptor modulators in chronic schizophrenia. CNS Drugs 25, 859-885 (2011).

34. Y. Chen, G. J. Guillemin, Kynurenine pathway metabolites in humans: disease and healthy States. Int J Tryptophan Res 2, 1-19 (2009).

35. C. Tapia-Rojas et al., Is L-methionine a trigger factor for Alzheimer's-like neurodegeneration?: Changes in $A \beta$ oligomers, tau phosphorylation, synaptic proteins, Wnt signaling and behavioral impairment in wild-type mice. Mol Neurodegener 10, 62-62 (2015).

36. Q. Wang, D. Liu, P. Song, M.-H. Zou, Tryptophan-kynurenine pathway is dysregulated in inflammation, and immune activation. Frontiers in bioscience (Landmark edition) 20, 11161143 (2015).

37. K. Sas, E. Szabó, L. Vécsei, Mitochondria, Oxidative Stress and the Kynurenine System, with a Focus on Ageing and Neuroprotection. Molecules 23, 191 (2018).

38. F. Fazio et al., Vasorelaxing Action of the Kynurenine Metabolite, Xanthurenic Acid: The Missing Link in Endotoxin-Induced Hypotension? Front Pharmacol 8, 214 (2017).

39. Y. Shi, Z. Liu, Y. Shen, H. Zhu, A Novel Perspective Linkage Between Kidney Function and Alzheimer's Disease. Front Cell Neurosci 12, 384 (2018).

40. J. K. Haukka et al., Metabolomic Profile Predicts Development of Microalbuminuria in Individuals with Type 1 Diabetes. Scientific Reports 8, 13853 (2018).

41. M. M. Mielke et al., Plasma sphingomyelins are associated with cognitive progression in Alzheimer's disease. Journal of Alzheimer's disease : JAD 27, 259-269 (2011).

42. K. A. Jhang, J. S. Park, H. S. Kim, Y. H. Chong, Sulforaphane rescues amyloid- $\beta$ peptidemediated decrease in MerTK expression through its anti-inflammatory effect in human THP-1 macrophages. J Neuroinflammation 15, 75 (2018).

43. G. Tondo, D. Perani, C. Comi, TAM Receptor Pathways at the Crossroads of Neuroinflammation and Neurodegeneration. Dis Markers 2019, 2387614 (2019).

44. H. C. Chiang, L. Wang, Z. Xie, A. Yau, Y. Zhong, PI3 kinase signaling is involved in Abetainduced memory loss in Drosophila. Proc Natl Acad Sci U S A 107, 7060-7065 (2010).

45. L. Wang et al., Epidermal growth factor receptor is a preferred target for treating Amyloid- $\beta$ induced memory loss. Proceedings of the National Academy of Sciences 109, 16743 (2012). 
46. T. Owens, T. Renno, V. Taupin, M. Krakowski, Inflammatory cytokines in the brain: does the CNS shape immune responses? Immunol Today 15, 566-571 (1994).

47. T. Kordula et al., Oncostatin $M$ and the interleukin- 6 and soluble interleukin- 6 receptor complex regulate alpha1-antichymotrypsin expression in human cortical astrocytes. J Biol Chem 273, 4112-4118 (1998).

48. J. V. Castell et al., Interleukin-6 is the major regulator of acute phase protein synthesis in adult human hepatocytes. FEBS Lett 242, 237-239 (1989).

49. C. R. Abraham, D. J. Selkoe, H. Potter, Immunochemical identification of the serine protease inhibitor alpha 1-antichymotrypsin in the brain amyloid deposits of Alzheimer's disease. Cell 52, 487-501 (1988).

50. D. J. Selkoe, The molecular pathology of Alzheimer's disease. Neuron 6, 487-498 (1991).

51. R. Tu, H. M. Grover, L. P. Kotra, Peptidyl Arginine Deiminases and Neurodegenerative Diseases. Curr Med Chem 23, 104-114 (2016).

52. N. K. Acharya et al., Neuronal PAD4 expression and protein citrullination: possible role in production of autoantibodies associated with neurodegenerative disease. J Autoimmun 38, 369-380 (2012).

53. T. Hamaguchi et al., Association of a polymorphism of the transforming growth factor-beta1 gene with cerebral amyloid angiopathy. J Neurol Neurosurg Psychiatry 76, 696-699 (2005).

54. D. K. Lahiri, Y. W. Ge, Role of the APP promoter in Alzheimer's disease: cell type-specific expression of the beta-amyloid precursor protein. Ann N Y Acad Sci 1030, 310-316 (2004).

55. E. K. Luedecking, S. T. DeKosky, H. Mehdi, M. Ganguli, M. I. Kamboh, Analysis of genetic polymorphisms in the transforming growth factor-beta1 gene and the risk of Alzheimer's disease. Hum Genet 106, 565-569 (2000).

56. T. Burton, B. Liang, A. Dibrov, F. Amara, Transforming growth factor-beta-induced transcription of the Alzheimer beta-amyloid precursor protein gene involves interaction between the CTCF-complex and Smads. Biochem Biophys Res Commun 295, 713-723 (2002).

57. S. Lesné et al., Transforming growth factor-beta 1 potentiates amyloid-beta generation in astrocytes and in transgenic mice. J Biol Chem 278, 18408-18418 (2003).

58. H. Ehrenreich et al., A hematopoietic growth factor, thrombopoietin, has a proapoptotic role in the brain. Proceedings of the National Academy of Sciences of the United States of America 102, 862-867 (2005).

59. H. G. Woo, Y. Chang, D. R. Ryu, T. J. Song, Plasma Klotho concentration is associated with the presence, burden and progression of cerebral small vessel disease in patients with acute ischaemic stroke. PLoS One 14, e0220796 (2019).

60. G. Paroni et al., Klotho at the Edge of Alzheimer's Disease and Senile Depression. Mol Neurobiol 56, 1908-1920 (2019).

61. K. Yang, Z. Yang, X. Chen, W. Li, The significance of sialylation on the pathogenesis of Alzheimer's disease. Brain Research Bulletin 173, 116-123 (2021).

62. I. Mohri et al., Hematopoietic prostaglandin D synthase and DP1 receptor are selectively upregulated in microglia and astrocytes within senile plaques from human patients and in a mouse model of Alzheimer disease. J Neuropathol Exp Neurol 66, 469-480 (2007).

63. E. Tarkowski et al., Increased intrathecal levels of the angiogenic factors VEGF and TGF-beta in Alzheimer's disease and vascular dementia. Neurobiol Aging 23, 237-243 (2002).

64. C. C. Chao et al., Serum cytokine levels in patients with Alzheimer's disease. Clin Diagn Lab Immunol 1, 433-436 (1994).

65. D. R. Royall, R. F. Palmer, $\delta$ scores predict mild cognitive impairment and Alzheimer's disease conversions from nondemented states. Alzheimers Dement (Amst) 6, 214-221 (2017).

66. V. B. Gupta et al., Altered levels of blood proteins in Alzheimer's disease longitudinal study: Results from Australian Imaging Biomarkers Lifestyle Study of Ageing cohort. Alzheimers Dement (Amst) 8, 60-72 (2017). 
medRxiv preprint doi: https://doi.org/10.1101/2021.07.14.21260511; this version posted August 15, 2021. The copyright holder for this preprint (which was not certified by peer review) is the author/funder, who has granted medRxiv a license to display the preprint in perpetuity. It is made available under a CC-BY-NC-ND 4.0 International license .

67. R. Taliyan, S. K. Chandran, V. Kakoty, Therapeutic Approaches to Alzheimer's Type of Dementia: A Focus on FGF21 Mediated Neuroprotection. Curr Pharm Des 25, 2555-2568 (2019).

68. G. Brombo et al., Lower Plasma Klotho Concentrations Are Associated with Vascular Dementia but Not Late-Onset Alzheimer's Disease. Gerontology 64, 414-421 (2018).

69. A. Salminen, P. Jouhten, T. Sarajärvi, A. Haapasalo, M. Hiltunen, Hypoxia and GABA shunt activation in the pathogenesis of Alzheimer's disease. Neurochem Int 92, 13-24 (2016).

70. Y. Hasin, M. Seldin, A. Lusis, Multi-omics approaches to disease. Genome Biol 18, 83 (2017).

71. M. Arif et al., iNetModels 2.0: an interactive visualization and database of multi-omics data. Nucleic Acids Res, (2021).

72. B. Hutter-Schmid, C. Humpel, Alpha-Smooth Muscle Actin mRNA and Protein Are Increased in Isolated Brain Vessel Extracts of Alzheimer Mice. Pharmacology 98, 251-260 (2016).

73. D. Åberg et al., Increased Cerebrospinal Fluid Level of Insulin-like Growth Factor-II in Male Patients with Alzheimer's Disease. J Alzheimers Dis 48, 637-646 (2015).

74. B. Yulug et al., Combined Metabolic Activators Improve Cognitive Functions without Altering Motor Scores in Parkinson's Disease. medRxiv, 2021.2007.2028.21261293 (2021).

75. S. Martire et al., Bioenergetic Impairment in Animal and Cellular Models of Alzheimer's Disease: PARP-1 Inhibition Rescues Metabolic Dysfunctions. J Alzheimers Dis 54, 307-324 (2016).

76. S. Martire, L. Mosca, M. d'Erme, PARP-1 involvement in neurodegeneration: A focus on Alzheimer's and Parkinson's diseases. Mech Ageing Dev 146-148, 53-64 (2015).

77. J. Le Douce et al., Impairment of Glycolysis-Derived I-Serine Production in Astrocytes Contributes to Cognitive Deficits in Alzheimer's Disease. Cell Metab 31, 503-517.e508 (2020).

78. R. Bavarsad Shahripour, M. R. Harrigan, A. V. Alexandrov, N-acetylcysteine (NAC) in neurological disorders: mechanisms of action and therapeutic opportunities. Brain Behav 4, 108-122 (2014).

79. G. Tardiolo, P. Bramanti, E. Mazzon, Overview on the Effects of N-Acetylcysteine in Neurodegenerative Diseases. Molecules 23, (2018).

80. Y. Hara, N. McKeehan, P. A. Dacks, H. M. Fillit, Evaluation of the Neuroprotective Potential of $\mathrm{N}$-Acetylcysteine for Prevention and Treatment of Cognitive Aging and Dementia. I Prev Alzheimers Dis 4, 201-206 (2017).

81. A. Kepka et al., Preventive Role of L-Carnitine and Balanced Diet in Alzheimer's Disease. Nutrients 12, 1987 (2020).

82. J. L. Flanagan, P. A. Simmons, J. Vehige, M. D. Willcox, Q. Garrett, Role of carnitine in disease. Nutr Metab (Lond) 7, 30-30 (2010).

83. A. Cristofano et al., Serum Levels of Acyl-Carnitines along the Continuum from Normal to Alzheimer's Dementia. PLoS One 11, e0155694 (2016).

84. N. Chen et al., L-carnitine for cognitive enhancement in people without cognitive impairment. Cochrane Database Syst Rev 3, CD009374-CD009374 (2017).

85. K. A. Wollen, Alzheimer's disease: the pros and cons of pharmaceutical, nutritional, botanical, and stimulatory therapies, with a discussion of treatment strategies from the perspective of patients and practitioners. Altern Med Rev 15, 223-244 (2010).

86. S. I. Gavrilova, B. Kalyn Ia, I. V. Kolykhalov, I. F. Roshchina, N. D. Selezneva, [Acetyl-L-carnitine (carnicetine) in the treatment of early stages of Alzheimer's disease and vascular dementia]. Zh Nevrol Psikhiatr Im S S Korsakova 111, 16-22 (2011).

87. K. K. Ting, B. Brew, G. Guillemin, The involvement of astrocytes and kynurenine pathway in Alzheimer's disease. Neurotox Res 12, 247-262 (2007).

88. K. O'Farrell, A. Harkin, Stress-related regulation of the kynurenine pathway: Relevance to neuropsychiatric and degenerative disorders. Neuropharmacology 112, 307-323 (2017).

89. G. J. Guillemin, G. A. Smythe, L. A. Veas, O. Takikawa, B. J. Brew, A beta 1-42 induces production of quinolinic acid by human macrophages and microglia. Neuroreport 14, 23112315 (2003). 
90. W. Wu et al., Expression of tryptophan 2,3-dioxygenase and production of kynurenine pathway metabolites in triple transgenic mice and human Alzheimer's disease brain. PLoS One 8, e59749 (2013).

91. A. Bryll et al., Oxidative-Antioxidant Imbalance and Impaired Glucose Metabolism in Schizophrenia. Biomolecules 10, (2020).

92. S. Seshadri et al., Plasma homocysteine as a risk factor for dementia and Alzheimer's disease. N Engl J Med 346, 476-483 (2002).

93. J. W. D. Griffin, P. C. Bradshaw, Amino Acid Catabolism in Alzheimer's Disease Brain: Friend or Foe? Oxid Med Cell Longev 2017, 5472792-5472792 (2017).

94. T. T. Braga et al., Soluble Uric Acid Activates the NLRP3 Inflammasome. Scientific reports 7, 39884-39884 (2017).

95. F. Hansmannel et al., Is the urea cycle involved in Alzheimer's disease? I Alzheimers Dis 21, 1013-1021 (2010).

96. V. Chouraki et al., Association of amine biomarkers with incident dementia and Alzheimer's disease in the Framingham Study. Alzheimers Dement 13, 1327-1336 (2017).

97. S. F. Graham et al., Untargeted metabolomic analysis of human plasma indicates differentially affected polyamine and L-arginine metabolism in mild cognitive impairment subjects converting to Alzheimer's disease. PLoS One 10, e0119452 (2015).

98. G. Wang et al., Plasma metabolite profiles of Alzheimer's disease and mild cognitive impairment. J Proteome Res 13, 2649-2658 (2014).

99. H. Chew, V. A. Solomon, A. N. Fonteh, Involvement of Lipids in Alzheimer's Disease Pathology and Potential Therapies. Frontiers in physiology 11, 598-598 (2020).

100. V. van der Velpen et al., Systemic and central nervous system metabolic alterations in Alzheimer's disease. Alzheimer's Research \& Therapy 11, 93 (2019).

101. D. Li et al., Prospective associations of plasma phospholipids and mild cognitive impairment/dementia among African Americans in the ARIC Neurocognitive Study. Alzheimers Dement (Amst) 6, 1-10 (2016).

102. X. Han et al., Metabolomics in early Alzheimer's disease: identification of altered plasma sphingolipidome using shotgun lipidomics. PLoS One 6, e21643 (2011).

103. D. Li et al., Plasma phospholipids and prevalence of mild cognitive impairment and/or dementia in the ARIC Neurocognitive Study (ARIC-NCS). Alzheimers Dement (Amst) 3, 73-82 (2016).

104. D. Ciavardelli et al., Medium-chain plasma acylcarnitines, ketone levels, cognition, and gray matter volumes in healthy elderly, mildly cognitively impaired, or Alzheimer's disease subjects. Neurobiol Aging 43, 1-12 (2016).

105. H. Oberacher et al., Targeted Metabolomic Analysis of Soluble Lysates from Platelets of Patients with Mild Cognitive Impairment and Alzheimer's Disease Compared to Healthy Controls: Is PC aeC40:4 a Promising Diagnostic Tool? J Alzheimers Dis 57, 493-504 (2017).

106. B. N. Simpson et al., Blood metabolite markers of cognitive performance and brain function in aging. J Cereb Blood Flow Metab 36, 1212-1223 (2016).

107. L. Whiley et al., Evidence of altered phosphatidylcholine metabolism in Alzheimer's disease. Neurobiol Aging 35, 271-278 (2014).

108. G. Paglia et al., Unbiased Metabolomic Investigation of Alzheimer's Disease Brain Points to Dysregulation of Mitoch ondrial Aspartate Metabolism. J Proteome Res 15, 608-618 (2016).

109. K. J. Castor et al., Urine dicarboxylic acids change in pre-symptomatic Alzheimer's disease and reflect loss of energy capacity and hippocampal volume. PLoS One 15, e0231765 (2020).

110. N. Kurbatova et al., Urinary metabolic phenotyping for Alzheimer's disease. Scientific reports 10, 21745-21745 (2020).

111. S. Passi et al., Saturated dicarboxylic acids as products of unsaturated fatty acid oxidation. Biochimica et Biophysica Acta (BBA) - Lipids and Lipid Metabolism 1168, 190-198 (1993).

112. V. Birzniece et al., Neuroactive steroid effects on cognitive functions with a focus on the serotonin and GABA systems. Brain Res Rev 51, 212-239 (2006). 
medRxiv preprint doi: https://doi.org/10.1101/2021.07.14.21260511; this version posted August 15, 2021. The copyright holder for this preprint (which was not certified by peer review) is the author/funder, who has granted medRxiv a license to display the preprint in perpetuity.

It is made available under a CC-BY-NC-ND 4.0 International license .

113. Y. Wang et al., Bile acids regulate cysteine catabolism and glutathione regeneration to modulate hepatic sensitivity to oxidative injury. JCl Insight 3, e99676 (2018).

114. G. L. Bowman et al., Ascorbic acid and rates of cognitive decline in Alzheimer's disease. J Alzheimers Dis 16, 93-98 (2009).

115. J. Milbrandt et al., Persephin, a Novel Neurotrophic Factor Related to GDNF and Neurturin. Neuron 20, 245-253 (1998).

116. K. B. Zihlmann et al., The GDNF family members neurturin, artemin and persephin promote the morphological differentiation of cultured ventral mesencephalic dopaminergic neurons. Brain Res Bull 68, 42-53 (2005).

117. C. Peña-Bautista et al., New screening approach for Alzheimer's disease risk assessment from urine lipid peroxidation compounds. Scientific Reports 9, 14244 (2019).

118. C. Proto et al., Plasma levels of neuropeptides in Alzheimer's disease. Gynecol Endocrinol 22, 213-218 (2006).

119. J. Olazarán et al., A blood-based, 7-metabolite signature for the early diagnosis of Alzheimer's disease. J Alzheimers Dis 45, 1157-1173 (2015).

120. R. Savica et al., Plasma sphingolipid changes with autopsy-confirmed Lewy Body or Alzheimer's pathology. Alzheimers Dement (Amst) 3, 43-50 (2016).

121. M. E. Ritchie et al., limma powers differential expression analyses for RNA-sequencing and microarray studies. Nucleic Acids Res 43, e47 (2015).

122. M. I. Love, W. Huber, S. Anders, Moderated estimation of fold change and dispersion for RNA-seq data with DESeq2. Genome Biol 15, 550 (2014).

123. K. L. Howe et al., Ensembl 2021. Nucleic Acids Research 49, D884-D891 (2020).

124. L. Väremo, J. Nielsen, I. Nookaew, Enriching the gene set analysis of genome-wide data by incorporating directionality of gene expression and combining statistical hypotheses and methods. Nucleic Acids Res 41, 4378-4391 (2013).

125. R. Agren et al., The RAVEN toolbox and its use for generating a genome-scale metabolic model for Penicillium chrysogenum. PLoS Comput Biol 9, e1002980 (2013).

126. M. Kanehisa, Y. Sato, KEGG Mapper for inferring cellular functions from protein sequences. Protein Sci 29, 28-35 (2020).

127. L. J. Pellegrino, A. J. Cushman, A. S. Pellegrino, A stereotaxic atlas of the rat brain. (New York (N.Y.) : Plenum press, ed. 2nd ed. 1967, 1979). 
medRxiv preprint doi: https://doi.org/10.1101/2021.07.14.21260511; this version posted August 15, 2021. The copyright holder for this preprint

\section{Figure Legends}

Figure 1. Transcriptomic analysis of brain tissue samples in ROSMAP datasets. A) Differential expression analysis for Alzheimer's disease (AD) brain samples compared to healthy controls was performed. Differentially expressed genes (DEGs) were determined from gene expression values. DEGs with a p-value of $1 \times 10-10$ or smaller after BenjaminiHochberg adjustment were determined statistically significant. Each point represents one gene. Red, significantly upregulated genes; blue, significantly downregulated genes; grey, not significant. B) Functional enrichment analysis was performed. Gene set enrichment analysis was applied on the DEGs to determine upregulated and downregulated GO terms compared to controls. Colour scale indicates direction of enrichment and p-value after BenjaminiHochberg adjustment. Significance code: *, Adj.p< 0.05. C) Carbon metabolism pathway analysis. Alterations to pathways were inferred from reporter metabolite analysis. Key reactions and pathways linking reporter metabolites (red nodes) are shown. Reactions are simplified and arrows may represent multiple reactions. D) Reporter metabolite analysis. Statistics from DEG analysis were used to infer altered metabolites based on the iBrain2845, functional genome-scale metabolic model of brain. Asterisks indicate statistical significance based on Student's t-test. P value $<0.05$.

\section{Figure 2. Animal study}

A) Rat animal groups in in vivo experiments. Group $1(n=4)$ were fed with only regular Chow diet (CHOW) for 5 weeks; Group $2(n=4)$ were fed with $\mathrm{CHOW}$ and treated with streptozotocin (STZ). The remaining animals in Group $3(n=4)$ were fed with high fat diet (HFD) for 3 weeks and sacrificed to verify model development. Groups 4-10 were fed with HFD for 5 weeks. After 3 weeks, the animals in Groups 5-10 were treated with STZ and administered with individual or combined metabolic activators, including L-serine, NAC, LCAT and NR for 2 weeks. B) Heatmap shows FC based alterations of the clinical variables in the rat study groups. Asterisks indicate statistical significance based on Student's t-test. P value <0.05. TG, triglyceride; TC, total cholesterol; ALP, Alkaline phosphatase; AST, Aspartate aminotransferase; ALT, Alanine aminotransferase; HDL, high-density lipoprotein; LDL, low-density lipoprotein; LDH, Lactate dehydrogenase. C) Histopathological, immunohistochemical (Caspase 3) and immunofluorescence (8-OHdG and H2A.X) images of rat brain (right side) and liver tissue (left side). Slides evaluated by independent pathologist and immunopositivity scores were: Absent (-), very mild (+), mild (++), moderate (+++) and severe $(++++)$. 
medRxiv preprint doi: https://doi.org/10.1101/2021.07.14.21260511; this version posted August 15, 2021. The copyright holder for this preprint

\section{Figure 3. CMAs Improves ADAS-Cog scores and clinical parameters.}

A) Study design for testing the effects of CMAs in AD patients. B) Differences in ADAS-Cog scores in the CMA and placebo groups on Days 0, 28 and 84 are presented. Additionally, differences in ADAS-Cog scores were analysed by stratifying the patients into high and low levels of ADAS-Cog groups (> 20 ADAS-Cog is high, $\leq 20$ is low). As decreased ADAS-Cog score is the indicator of the improved cognitive function in AD patients, the ADAS-Cog scores is significantly decreased on Day 28 vs Day 0 (Log2FoldChange $(\mathrm{FC})=-0.33,(26 \%$ improvement $), \quad \mathrm{p}$-value $=0.0000003)$ and Day 84 vs Day $0 \quad(\log 2 \mathrm{FC}=-0.37, \quad(29 \%$ improvement), $\mathrm{p}$-value $=0.00001$ ) in the CMA group. A slightly but significant decrease is found in the placebo group on Day 28 vs Day $0(\log 2 \mathrm{FC}=-0.16,(12 \%$ improvement $), p$ value $=0.009)$ and Day 84 vs Day $0(\log 2 \mathrm{FC}=-0.19,(14 \%$ improvement $), p$-value $=0.001)$ due to the recommendations of exercise and Mediterranean diet to all AD patients participated in the trial. The differences between clinical parameters have also been analysed by stratifying the patients into low-scored (mild patients) and high-scored (severe patients) ADAS-Cog groups (>20ADAS-Cog score is high, $\leq 20$ is low). The ADAS-Cog scores is significantly decreased on Day 28 vs Day $0(\log 2 \mathrm{FC}=-0.31$, $(24 \%$ improvement $), \mathrm{p}$-value $=0.002)$ and Day 84 vs Day 0 (Log2FC=-0.38, (30\% improvement), p-value=0.003) in the high scored CMA group and no significance difference in the high-scored placebo ( $>0.05$ in both time points) group. C) We selected 10 patients from the severe (ADAS-COG > 20) CMA group with matched ADAS-COG values to the placebo group (P-Value: 0.693) and presented the ADAS-Cog scores. We recalculated the differences in ADAS-COG scores, and found significant improvement in the CMA group whereas no significant difference in the placebo group. D) Heatmaps shows $\log 2 \mathrm{FC}$ based alterations of the clinical variables, which are compared before and after the administration of CMA in both drug and placebo groups. Asterisks indicate statistical significance based on Student's t-test. P value <0.05. Log2FC: $\log 2$ (fold change).

\section{Figure 4. Identification of clinical variables informative for response to CMAs administration.}

A) Distribution of ADAS-Cog scores over visit number for patients with ALT $\leq 16$ IU/L at visit 1 (upper panel) and patients with ALT > $16 \mathrm{IU} / \mathrm{L}$ at visit 1 (lower panel). B) Betweenvisit changes to ADAS-Cog with various clinical variable groupings are shown. Only those clinical variable groupings resulting in a more significant change to ADAS-Cog in CMA 
medRxiv preprint doi: https://doi.org/10.1101/2021.07.14.21260511; this version posted August 15, 2021. The copyright holder for this preprint (which was not certified by peer review) is the author/funder, who has granted medRxiv a license to display the preprint in perpetuity.

It is made available under a CC-BY-NC-ND 4.0 International license .

group compared to placebo group (a p-value of 0.05 or better are shown). Colour scale indicates $\log 2$ fold change to ADAS-Cog between visits. Statistical significance between visits was determined by a paired t-test across individuals who attended both visits. Asterisks indicate statistical significance $\mathrm{p}<0.05$.

\section{Figure 5 CMAs alters plasma metabolite levels}

A) Differences in the plasma levels of individual CMAs including serine, carnitine, cysteine and nicotinamide are shown in the CMA and placebo groups on Days 0 and 84. Plasma level of B) amino acids, C) lipids and D) other metabolites that are significantly different between Day 84 vs Day 0 in the CMA and placebo groups are presented. Adj. $\mathrm{p}<0.05$. Heatmap shows $\log 2 \mathrm{FC}$ values of metabolites between Day 84 vs Day 0. Asterisks indicate statistical significance based on paired Student's t test. Adj.p< 0.05. Log2FC: $\log 2$ (fold change).

\section{Figure 6 Correlation of CMAs with plasma metabolites and altered plasma protein levels}

A) Associations between the plasma level of individual CMAs and the 10 most significantly correlated plasma metabolites are presented. Asterisks indicate statistical significance (Adj.p $<0.05)$ based on Spearman correlation analysis. Cor.Coeff: Correlation coefficient B) Heatmap shows $\log 2 \mathrm{FC}$ based alterations between the significantly different proteins on Day 84 vs Day 0 in the CMA and placebo groups. Asterisks indicate statistical significance based on paired Student's t test. $\mathrm{p}<0.01$. C) Integrated multi-omics data based on network analysis represents the neighbours of the CMAs, including serine, carnitine, nicotinamide and cysteine, and ADAS-Cog scores. Only analytes that are significantly altered in CMA Day 84 vs Day 0 are highlighted. 


\section{Supplementary datasets}

Dataset S1. Transcriptomic analysis of Alzheimer's disease brain tissue samples in ROSMAP datasets.

Dataset S2. Study groups, body weight of animals and statistical analysis results of animal study

Dataset S3. Collection of samples of CMA and placebo groups and the measured values of clinical indicators before and after treatment.

Dataset S4. Statistical analysis of clinical indicators between different visits or groups.

Dataset S5. Plasma metabolomics data for each patient before and after treatment and statistical analysis of plasma metabolites between different visits or groups.

Dataset S6. The association between the plasma level of the four supplements serine, carnitine, cysteine and nicotinamide riboside with the plasma levels of other metabolites.

Dataset S7. Plasma proteomics data generated with the Olink cardiometabolic, inflammation, neurology and oncology panels for each patient before and after treatment and statistical analysis of plasma proteins between different visits or groups.

Dataset S8. Multi-Omics Network Data, including edges and nodes information. The network is presented in the iNetModels (http://inetmodels.com). 
medRxiv preprint doi: https://doi.org/10.1101/2021.07.14.21260511; this version posted August 15, 2021. The copyright holder for this preprint (which was not certified by peer review) is the author/funder, who has granted medRxiv a license to display the preprint in perpetuity.

It is made available under a CC-BY-NC-ND 4.0 International license.

Table 1 Scoring histopathological, immunohistochemical and immunofluorescence findings in brain tissues and liver tissues

\begin{tabular}{|c|c|c|c|c|c|c|c|}
\hline Organ & Groups & Hyperemia & $\begin{array}{l}\text { Degeneration in } \\
\text { Neurons }\end{array}$ & $\begin{array}{l}\text { Necrosis in } \\
\text { Neurons }\end{array}$ & $\begin{array}{l}\text { 8-OHdG } \\
\text { (IHC) }\end{array}$ & $\begin{array}{l}\text { Caspase } 3 \\
\text { (IFA } \\
\text { FITC) }\end{array}$ & $\begin{array}{l}\text { H2A.X } \\
\text { (IFA } \\
\text { Texas } \\
\text { Red) }\end{array}$ \\
\hline \multirow{10}{*}{ BRAIN } & CHOW & - & - & - & - & - & - \\
\hline & CHOW + STZ & +++ & +++ & +++ & +++ & +++ & +++ \\
\hline & HFD 3w & +++ & +++ & +++ & +++ & +++ & +++ \\
\hline & HFD 5w & ++++ & ++++ & ++++ & ++++ & ++++ & ++++ \\
\hline & HFD+STZ & ++++ & ++++ & ++++ & ++++ & ++++ & ++++ \\
\hline & HFD+STZ+Serine & +++ & +++ & ++ & +++ & +++ & +++ \\
\hline & HFD+STZ+NAC & ++++ & ++++ & +++ & +++ & +++ & +++ \\
\hline & HFD+STZ+LCAT & +++ & +++ & ++ & +++ & +++ & +++ \\
\hline & HFD+STZ+NR & +++ & +++ & ++ & +++ & +++ & +++ \\
\hline & $\mathrm{HFD}+\mathrm{STZ}+$ Serine+NAC+LCAT+NR & ++ & ++ & + & ++ & ++ & ++ \\
\hline \multirow{10}{*}{ LIVER } & CHOW & - & - & - & - & - & - \\
\hline & CHOW + STZ & +++ & +++ & +++ & +++ & +++ & +++ \\
\hline & HFD 3w & +++ & +++ & +++ & +++ & +++ & +++ \\
\hline & HFD 5w & ++++ & ++++ & ++++ & ++++ & ++++ & ++++ \\
\hline & HFD+STZ & ++++ & ++++ & ++++ & ++++ & ++++ & ++++ \\
\hline & HFD+STZ+Serine & +++ & +++ & +++ & +++ & +++ & +++ \\
\hline & $\mathrm{HFD}+\mathrm{STZ}+\mathrm{NAC}$ & +++ & ++++ & +++ & +++ & +++ & +++ \\
\hline & HFD+STZ+LCAT & +++ & +++ & +++ & +++ & +++ & +++ \\
\hline & HFD+STZ+NR & +++ & +++ & ++ & +++ & +++ & +++ \\
\hline & HFD+STZ+Serine+NAC+LCAT+NR & +++ & +++ & + & + & ++ & ++ \\
\hline
\end{tabular}




\section{Figure 1}

It is made available under a CC-BY-NC-ND 4.0 International license .

A

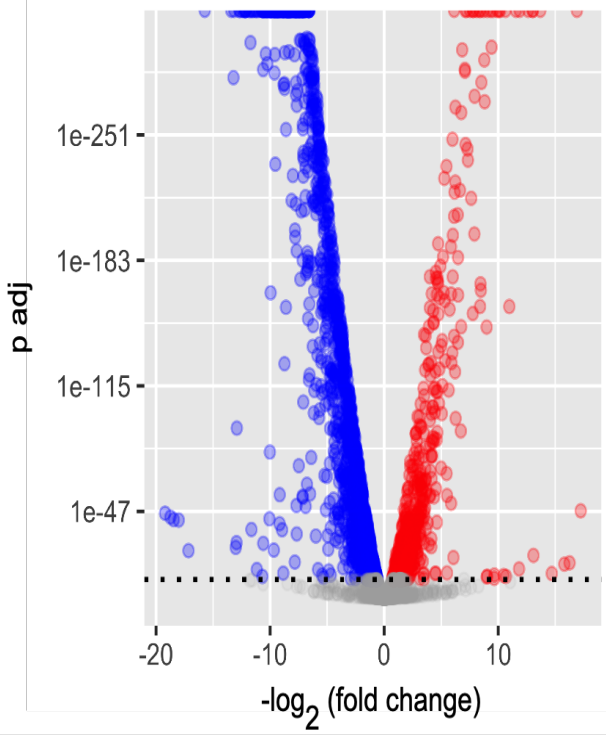

B

RNA polymerase II cis-regulatory region sequence-specific DNA bi response to organic cyclic compound regulation of transcription, DNA-templated regulation of gene expressionprotein-containing complex assembly protein-containing complex protein heterodimerization activity protein dimerization activity protein C-terminus binding positive regulation of transcription by RNA polymerase 1 positive regulation of cell population proliferation perinuclear regiona membrane ossification nucleotide binding nucleoplasm nucleic acid binding nuclear chromatin nervous system development negative regulation of transcription, DNA-templated negative regulation of transcription by RNA polymerase IInegative regulation of cell population proliferation multicellular organism development metal ion binding membrane -
lipid binding intracellular protein transpoit intracellular membrane-bounded orcanelleidentical protein binding growth factor activity ocal adhesion extracellular space extracellular space extracellular region -
extracellular matrix structural constituent extracellular matrix cexporome enzyme binding -
endosome endoplasmic reticulum DNA-binding transcription factor activity, RNA polymerase ll-speDNA-binding transcription activator activity, RNA polvmerase IIDNA binding cytosol-
cytoskeleton cytoplasmic vesicle membrane collagen-containing extracellular matrixchromosomechromosome-
cell surfacecell projection $-\log _{10}(p$ adj $)$ Downregulated | Upregulated $\begin{array}{lll}3 & 0 & 3\end{array}$ cell differentiation catalytic activity Golgi apparatus -

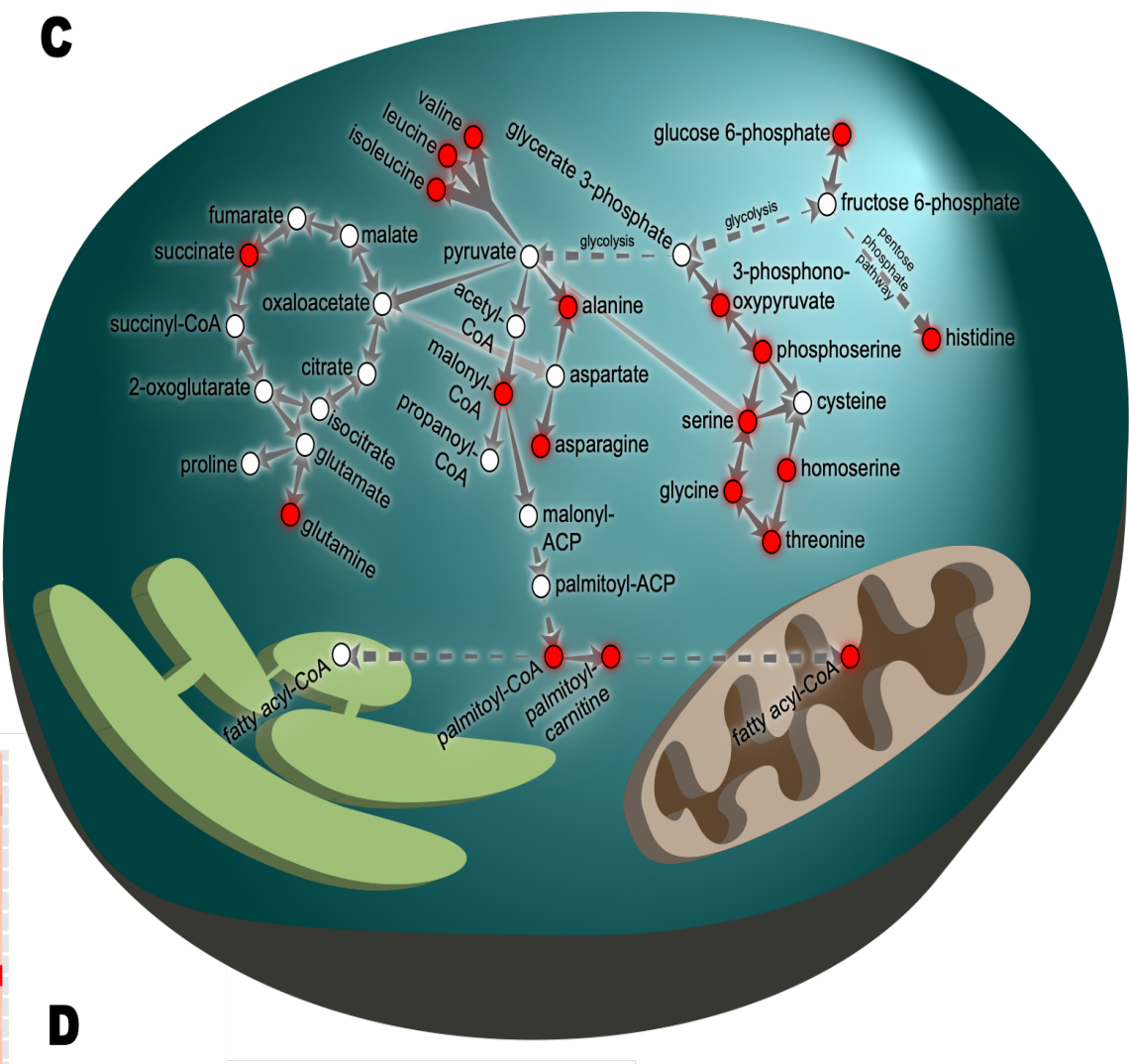

D

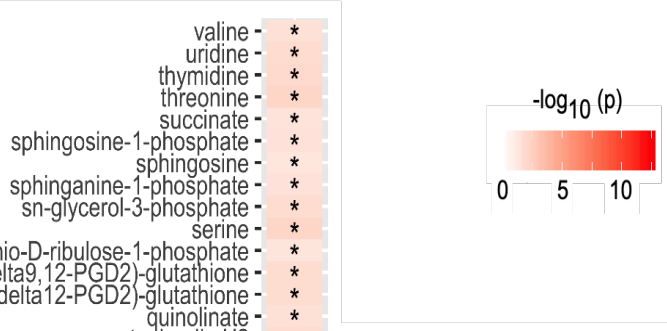

S-(11-OH-9-deoxy-dhio-D-ribulose-1-phosphate S-(11-hydroxy-9-deoxy-delta12-PGD2)-glutathione prostaglandin $\mathrm{H} 2$ procollagen-5-hydroxy-L-lysinepalmitoleoyl-carnitine -

N1,N12-diacetylspermine $\mathrm{N}$-formimino-L-glutamate $\mathrm{N}$-acetyl-D-mannosamine mannosemalonyl-COA-" malony-carnitin malonic-dialdehyde -
linoleic-carnitinelinoleic-carnitine -
leucine lauroyl-COA isoleucine inositol homoserine histidine histamine -
HCO3-guanosine glycine glucose-6-phosphate glucosamine fatty acids (22) dihydrolipoamide cysteamine asparagine arachidonyl-carnitine adenylyl sulfateacylcarnitine $(60)$ acyl-CoA (270)activation-ppara acetamidopropanal cell body cell adhesion calcium ion binding ATP bindingapical part of cell actin filament binding actin cytoskeleton
actin binding $5,10-$ methenyl-
5 -formiminotetrahydrofolate -
syloxy)-L-lysine-procollagen 4-aminobutyrate 3-phosphonooxypyruvate 3-phosphonooxypyruvate 2-deoxy-D-ribose-5-phosphate 1D-myo-inositol-3-phosphate 12(S)-HHT -

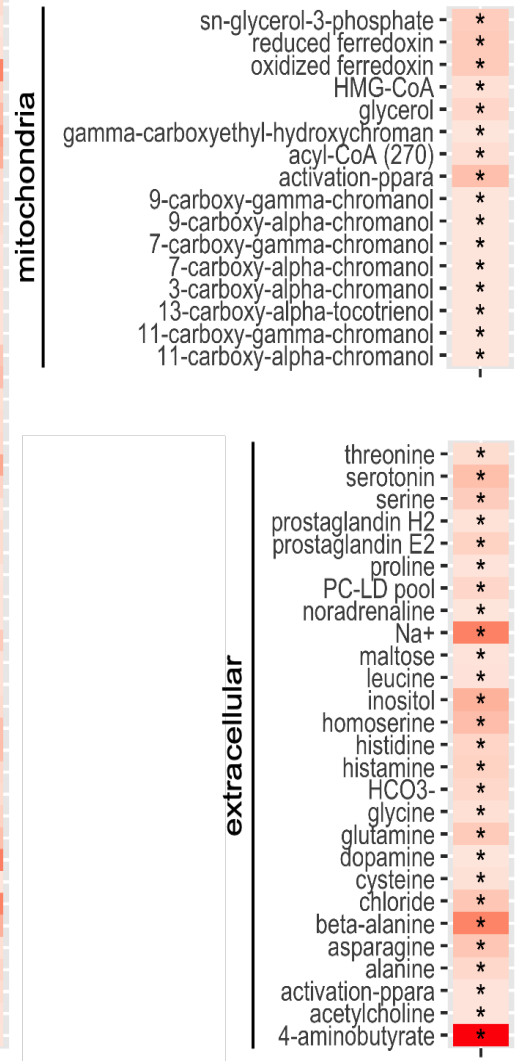




\section{Figure 2}

A

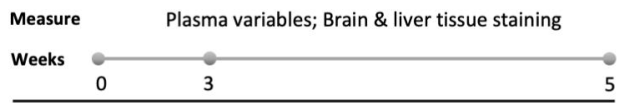

\begin{tabular}{ccccccc}
\hline Group & Diet & STZ & Serine & NAC & LCAT & NR \\
\hline $1(n=4)$ & Chow diet & & & & & \\
$2(n=4)$ & Chow diet & $\checkmark$ & & & & \\
$3(n=4)$ & HFD & & & & & \\
$4(n=4)$ & HFD & & & & & \\
$5(n=3)$ & HFD & $\checkmark$ & & & & \\
$6(n=4)$ & HFD & $\checkmark$ & $\checkmark$ & & & \\
$7(n=3)$ & HFD & $\checkmark$ & & $\checkmark$ & & \\
$8(n=4)$ & HFD & $\checkmark$ & & & $\checkmark$ & $\checkmark$ \\
$9(n=4)$ & HFD & $\checkmark$ & & & & $\checkmark$ \\
$10(n=3)$ & HFD & $\checkmark$ & $\checkmark$ & $\checkmark$ & $\checkmark$ & $\checkmark$ \\
\hline
\end{tabular}

2 weeks CMAs Administration (individual or combination)

\section{B}

(1)
Fold change

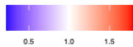

Group 5 vs Group 4 -
Group 6 vs Group 4 -
Group 7 vs Group 4 -
Group 8 vs Group 4 -
Group 9 vs Group 4 -
Group 10 vs Group 4 -

एक

\section{Liver tissue staining}

C
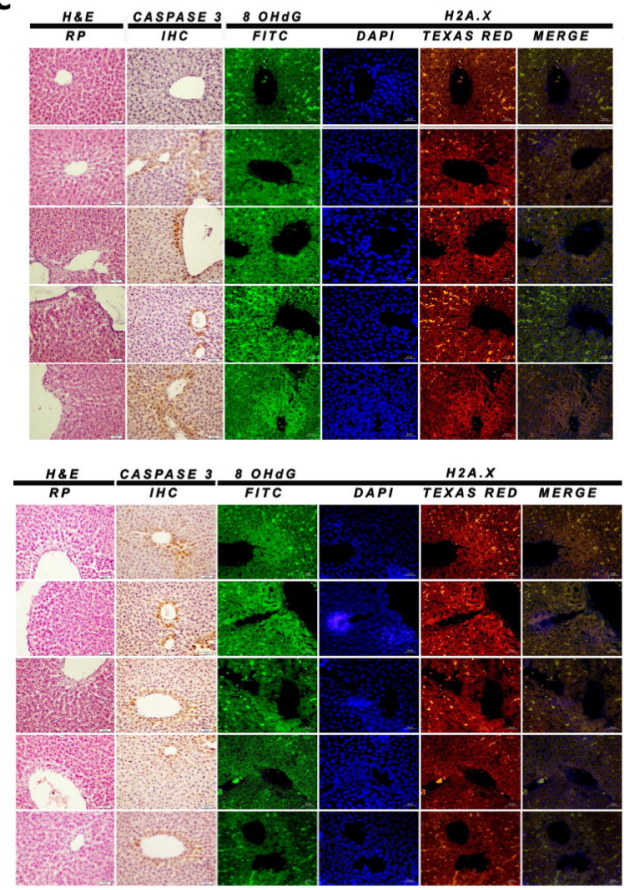

\section{Brain tissue staining}
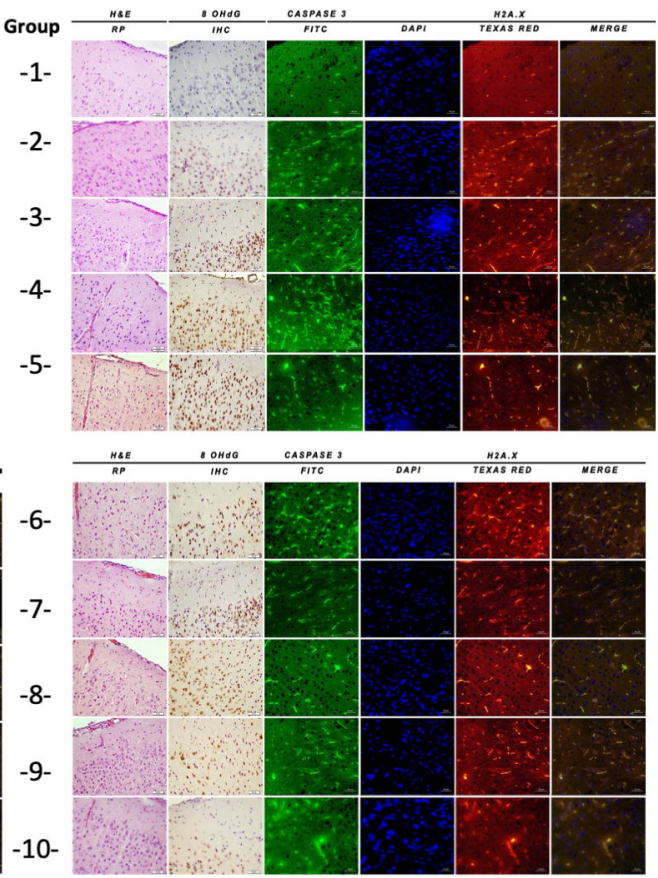


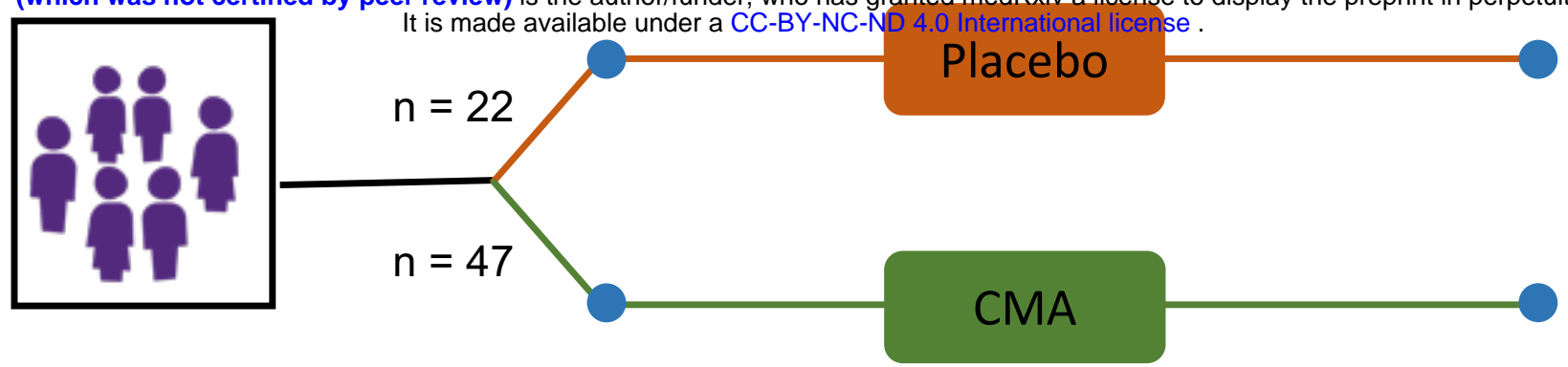

Timeline

Screening

$(n=89)$

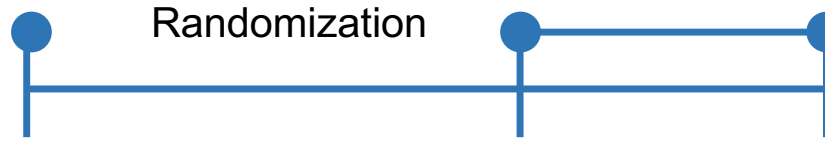

Visit 1: day_0 Visit 2: day_28

$(n=69) \quad(n=69)$

Clinical parameters, Metabolomics \& Proteomics

\section{Measure}

B

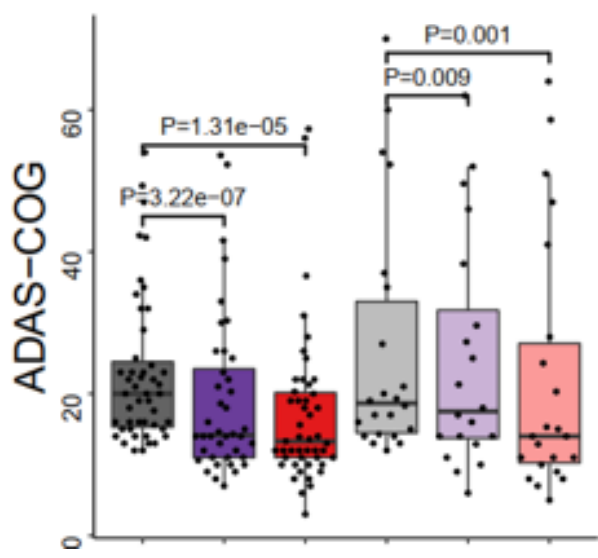

Visit 1 Visit 2 Visit 3 Visit 1 Visit 2 Visit 3 CMA

Placebo

C
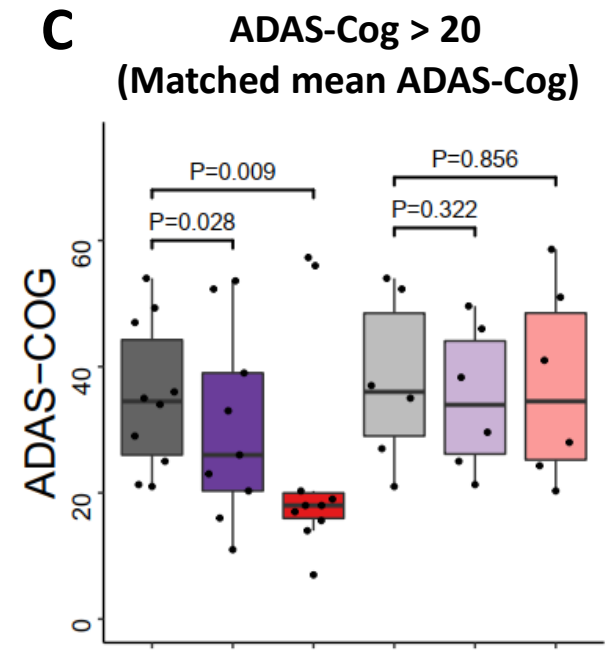

Visit 1 Visit 2 Visit 3 Visit 1 Visit 2 Visit 3 CMA Placebo

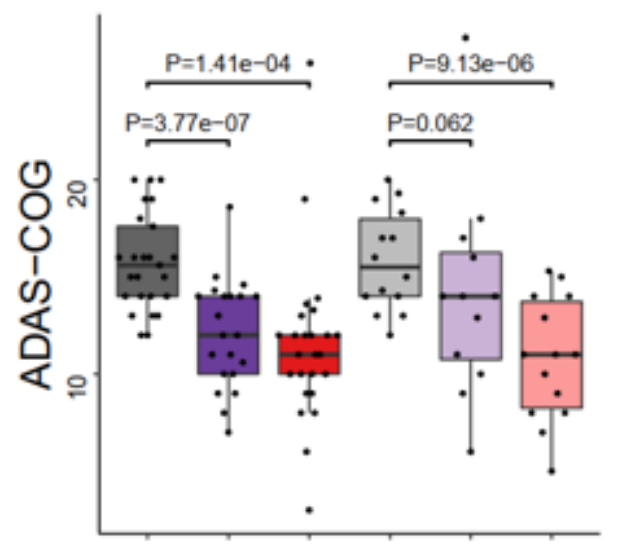

Visit 1 Visit 2 Visit 3 Visit 1 Visit 2 Visit 3

CMA

Placebo

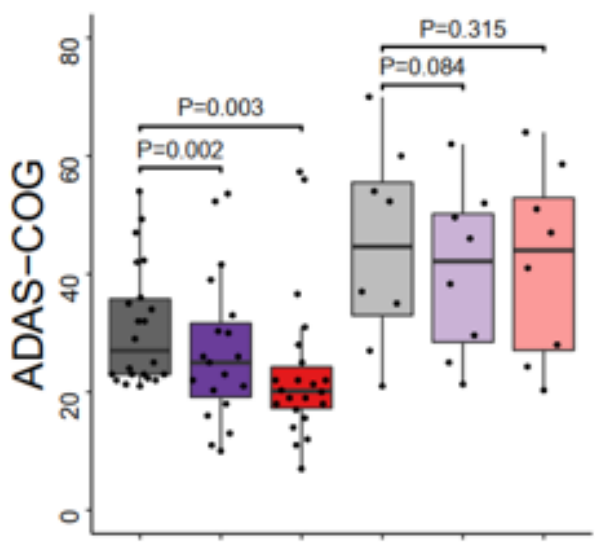

Visit 1 Visit 2 Visit 3 Visit 1 Visit 2 Visit 3

CMA
Visit 3: day_84

$(n=60)$

\section{D}

All patients
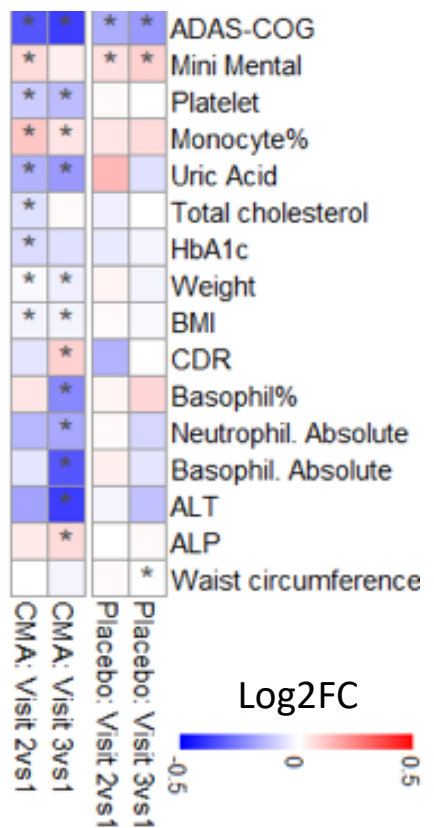

ADAS-Cog $\leq \mathbf{2 0}$

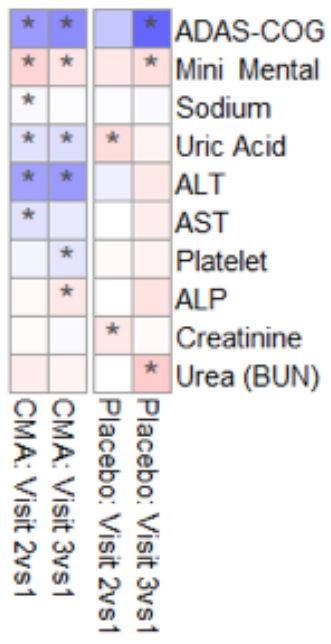

$\log 2 \mathrm{FC}$
ADAS-Cog $>20$

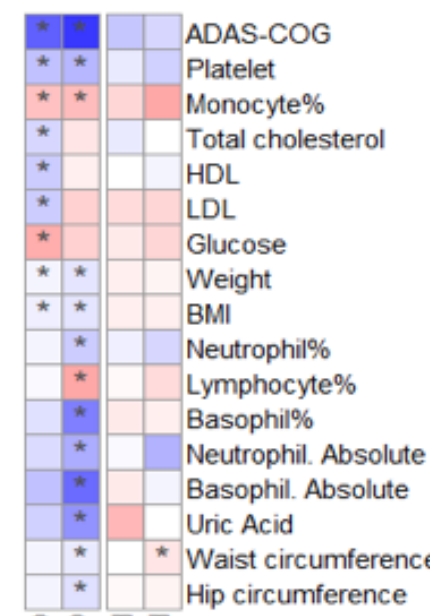

$? ? \frac{0}{0}$ $\leq \leq \%$ Log2FC 
$\underline{\text { Visit } 1 \text { Visit } 3 \quad \text { Visit } 1 \text { Visit } 3}$

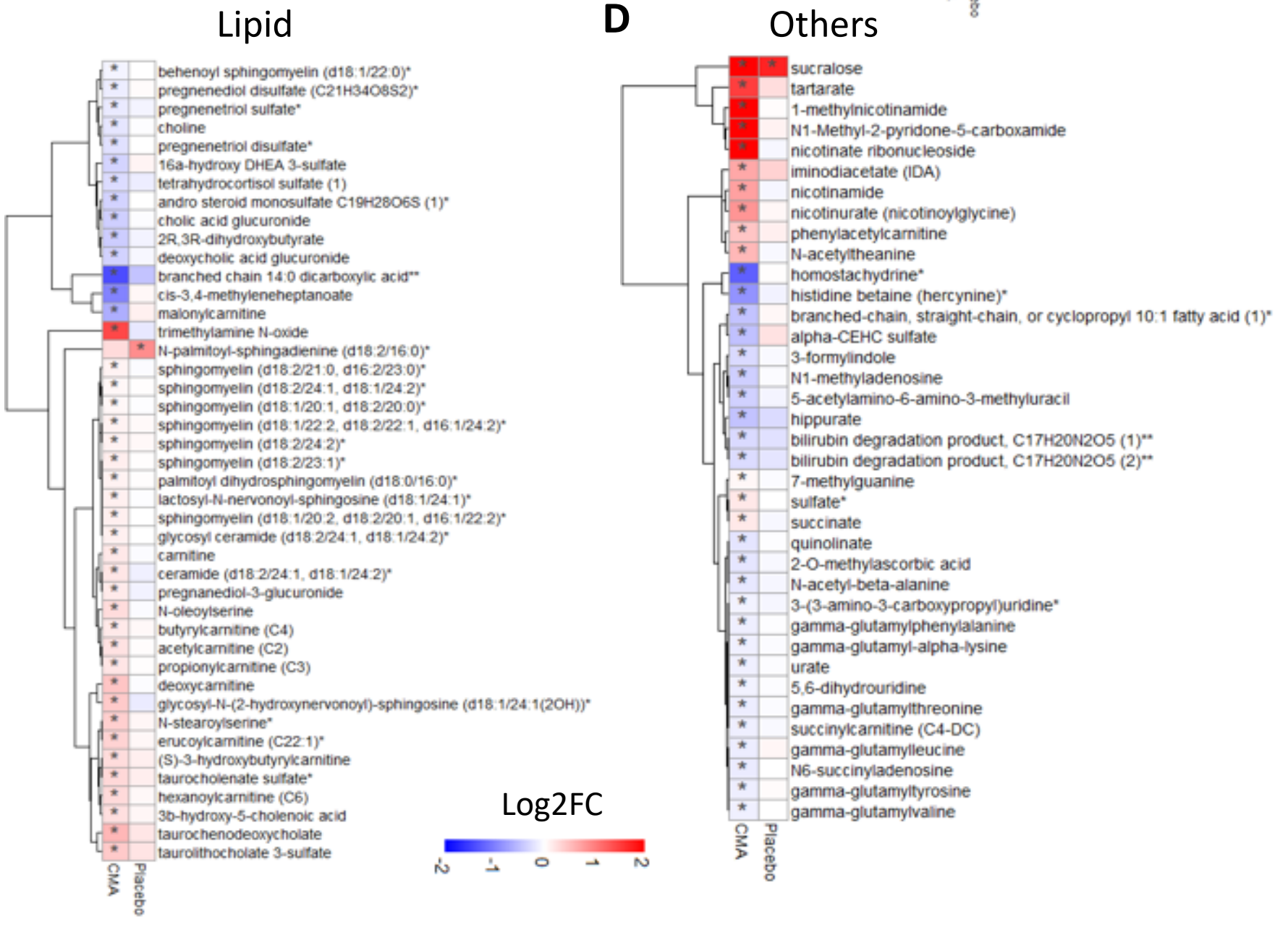

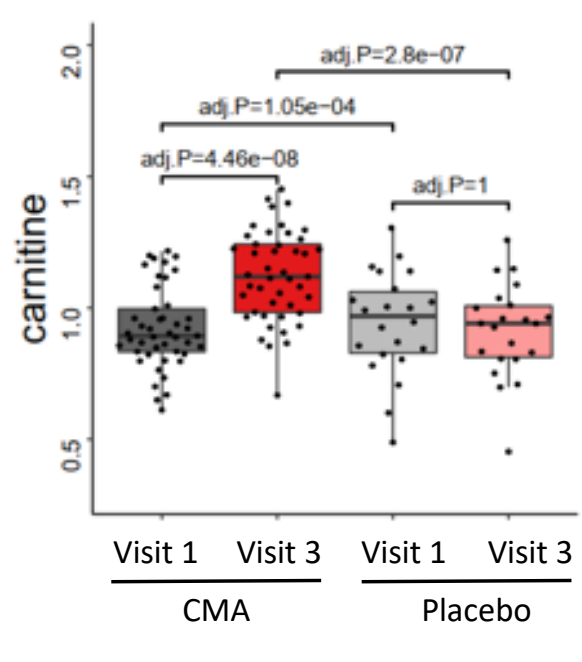

B

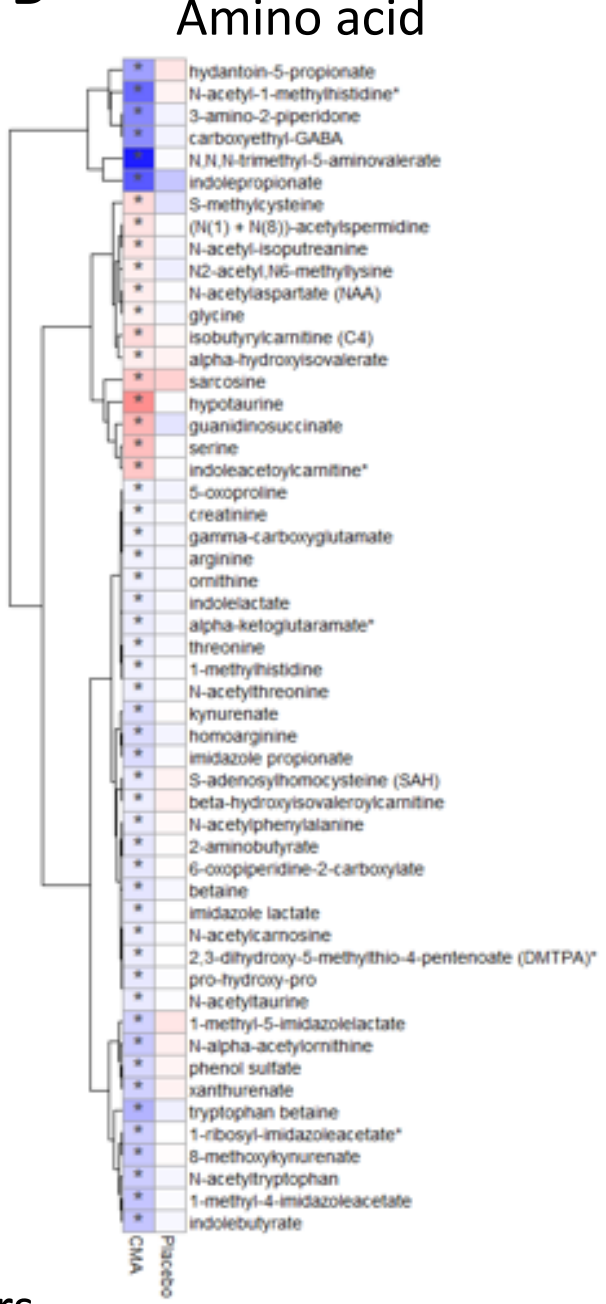

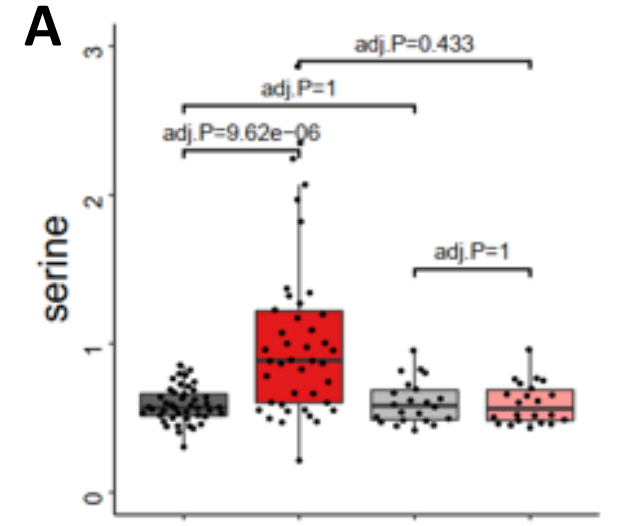

Visit 1 Visit 3 Visit 1 Visit 3 CMA Placebo

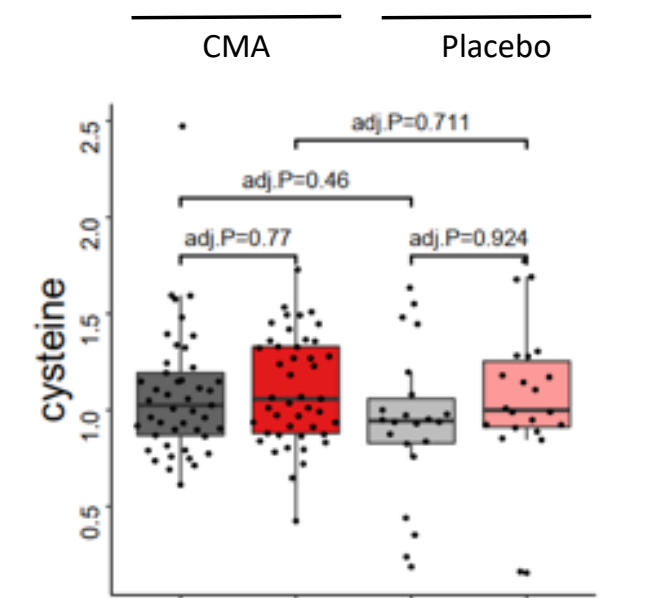

C

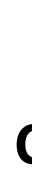

CMA Placebo

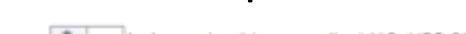
choine

charylic acid"

N-palmiloy-sphingatienine

(d

palmitoyl dinydrosphingomvelin (d18:0/16:0)"

actosyl-N-nervonoyl-sphingosine (d18:1/24.1)

camnine

ceramide (d182/24:1, $118: 1 / 24-2)^{*}$

pegnanediol-3-giucuronice

butyryicarntine

(C3) sphingomyelin (d18:1/20:2, d18:2/20:1, d16:1/22:2)

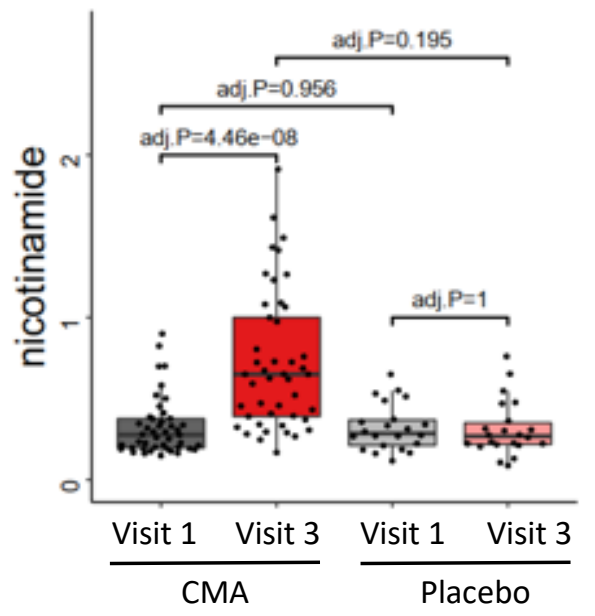

Placebo

\section{Others}

r review) is the author/funder, who has granted medRxiv a license to display the preprint in perpetuit 
Figure 6

A

It is made available under a CC-BY-NC-ND 4.0 International license.

Cor.coef

\begin{tabular}{|c|c|c|c|c|c|c|c|c|c|c|c|c|c|c|c|c|c|c|c|c|c|c|c|c|c|c|c|}
\hline & & & ᄃ & & & & & & & ש & & & & & & & & & & & & & & & & & \multirow{3}{*}{$\begin{array}{l}\text { serine } \\
\text { carnitine }\end{array}$} \\
\hline * & * & * & * & * & ; & * & * & * & * & * & * & & & * & * & t & * & t & & & & & & & & & \\
\hline * & * & * & * & * & * & * & * & * & 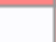 & $\star$ & $\star$ & ᄎ & * & * & * & $\star$ & & & & & $\hbar$ & & & & & & \\
\hline & & & & & & & & & & & & & * & & & & & * & * & * & * & * & * & * & $\star$ & * & cysteine \\
\hline k & $\star$ & * & * & * & * & * & * & * & * & * & & * & & * & * & & * & & & & & & & & & & nicotinamide \\
\hline
\end{tabular}

B

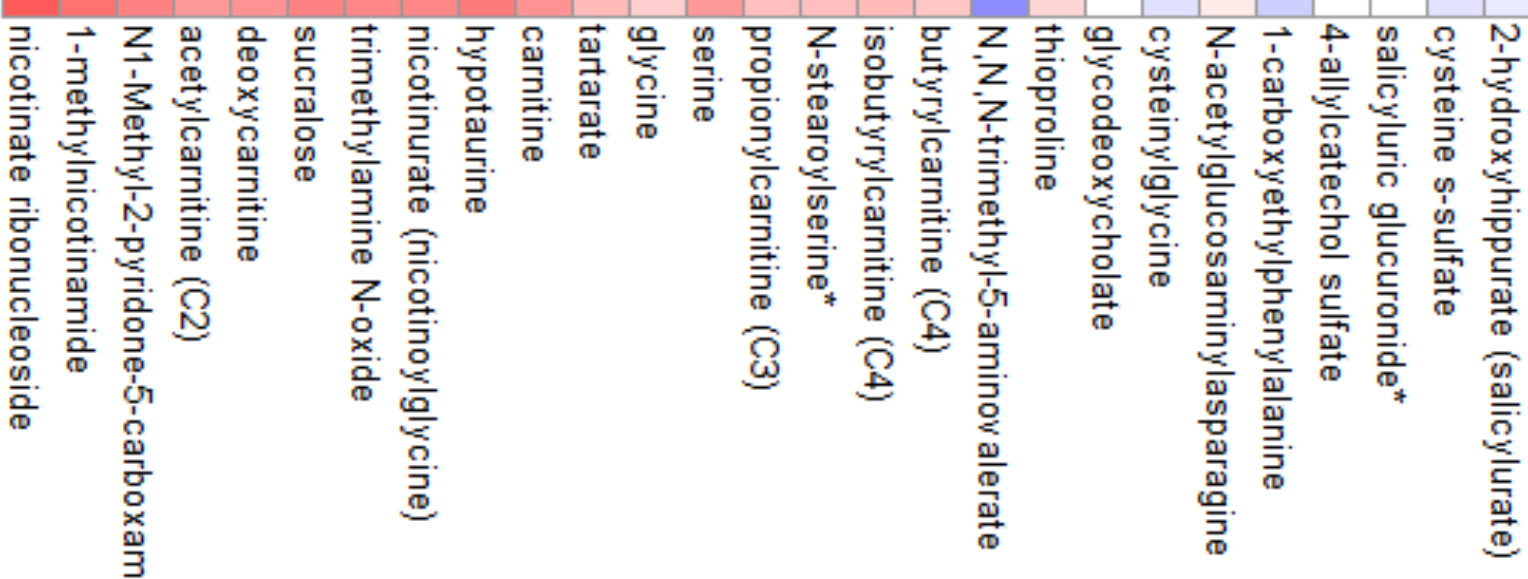

$\log 2 \mathrm{FC}$

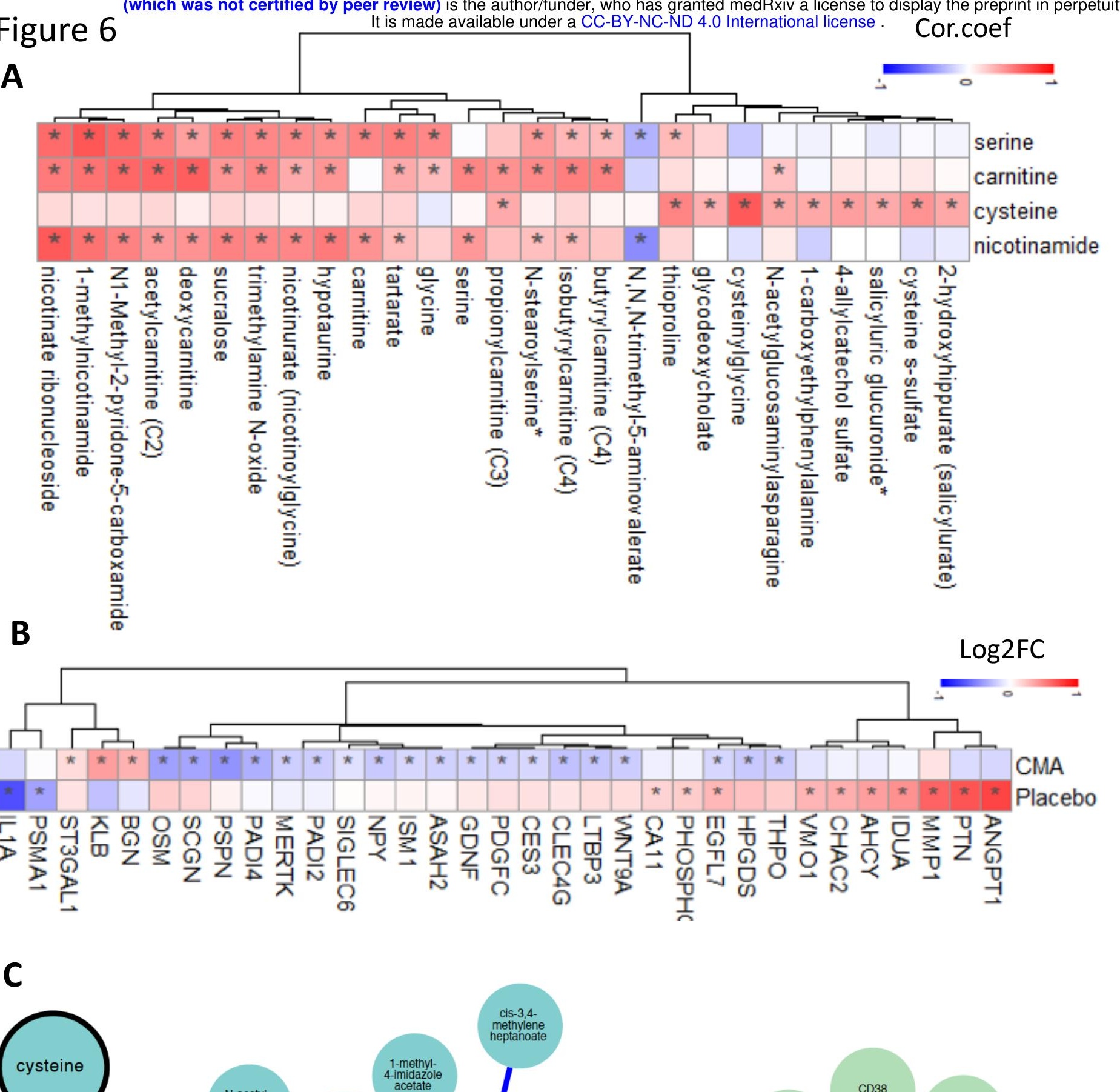

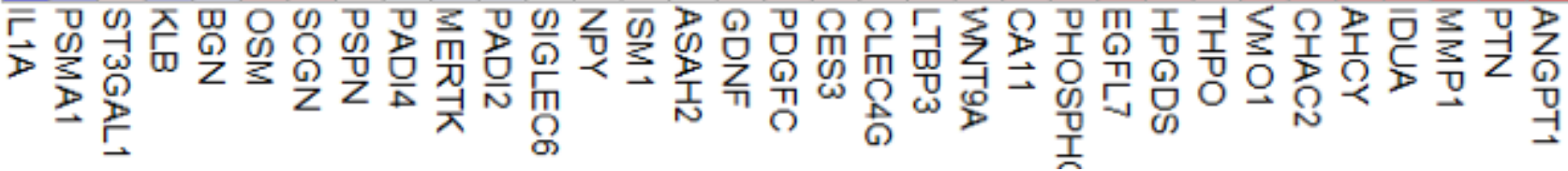

C
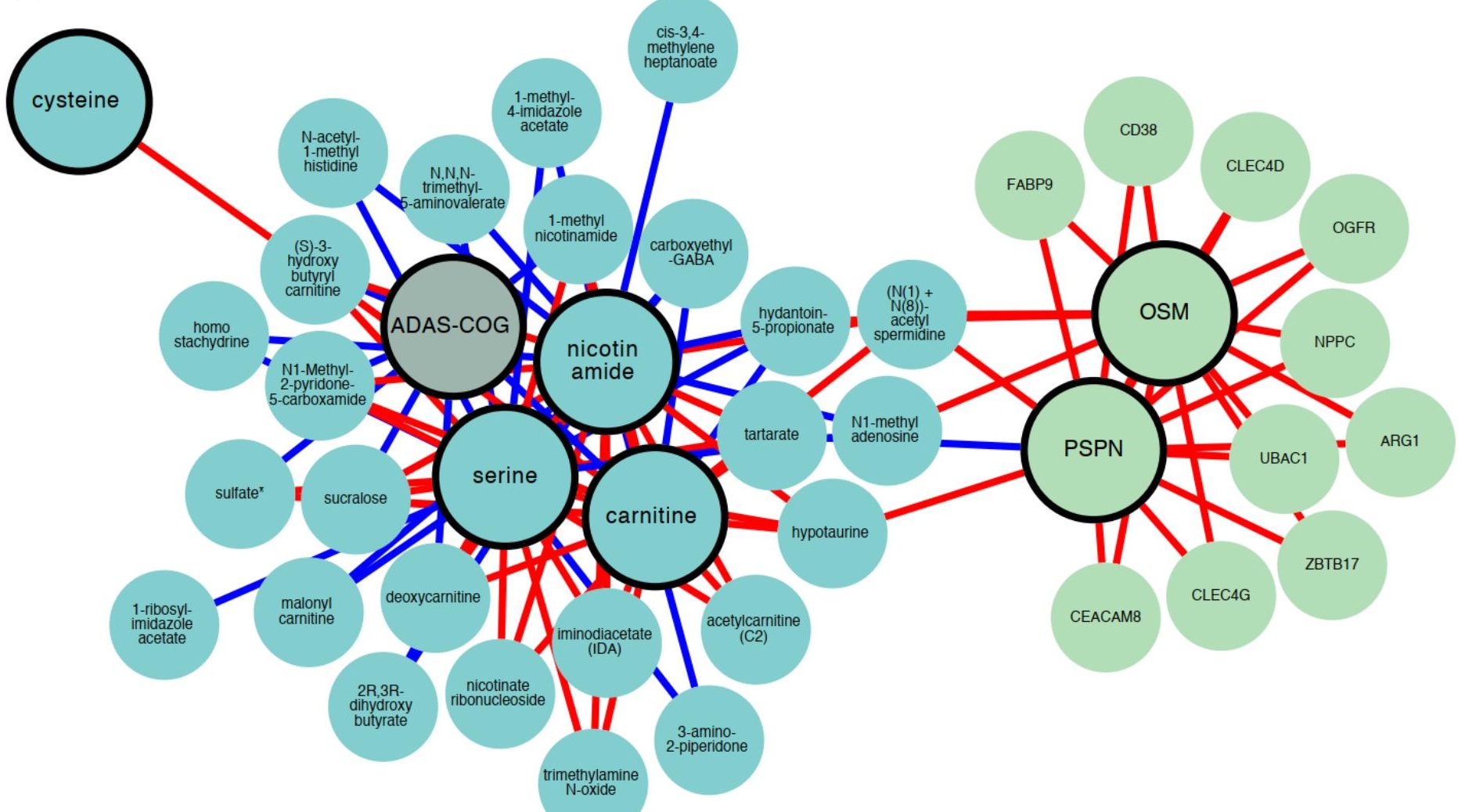

cis-3,4-

eptanoate

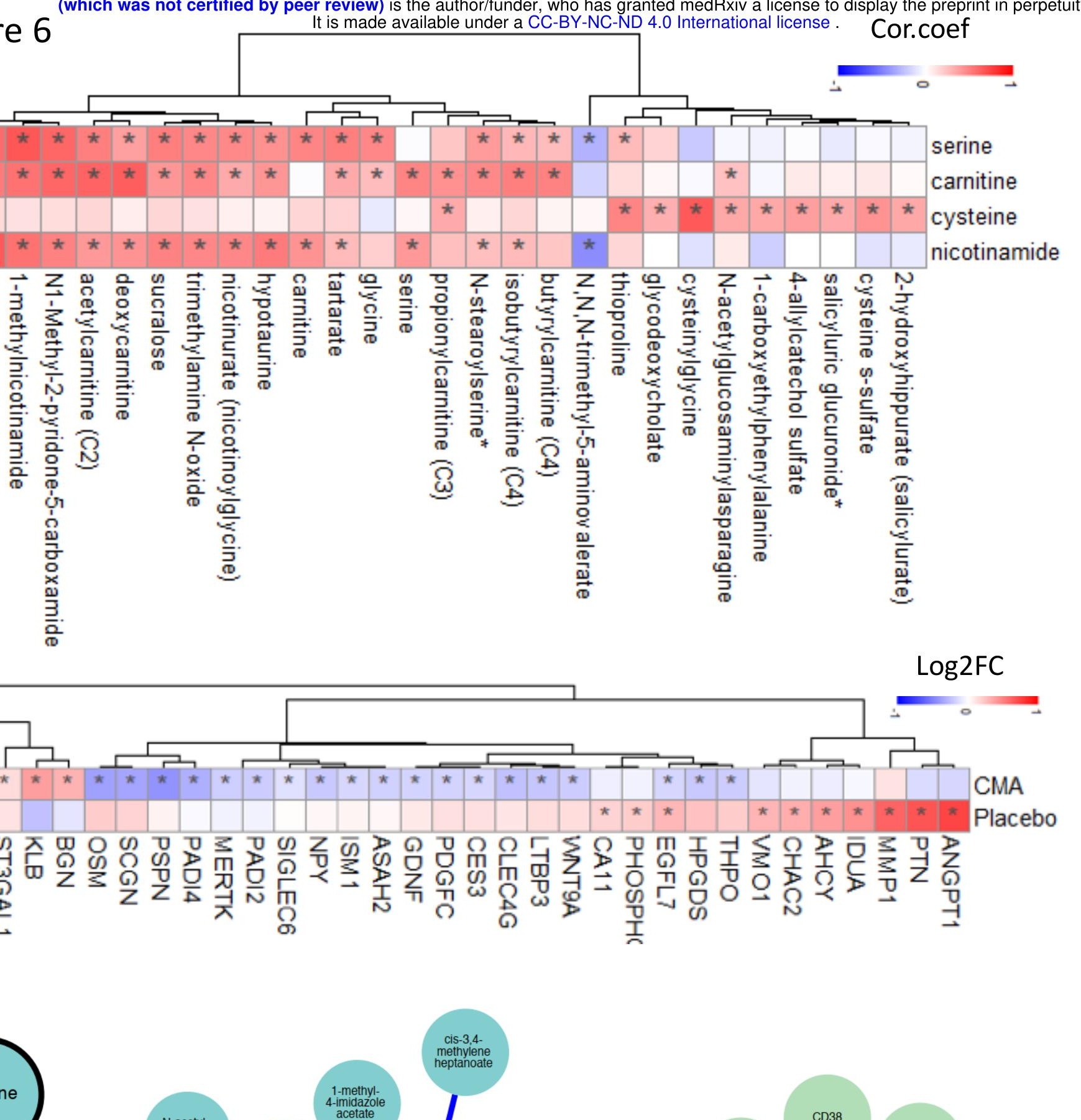

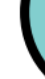

